This is a self-archived version of an original article. This version may differ from the original in pagination and typographic details.

Author(s): Geiss, Christel; Labart, Céline; Luoto, Antti

Title: Random walk approximation of BSDEs with Hölder continuous terminal condition

Year: 2020

Version: Published version

Copyright: (C) 2020 ISI/BS

Rights: In Copyright

Rights url: http://rightsstatements.org/page/lnC/1.0/?language=en

Please cite the original version:

Geiss, C., Labart, C., \& Luoto, A. (2020). Random walk approximation of BSDEs with Hölder continuous terminal condition. Bernoulli, 26(1), 159-190. https://doi.org/10.3150/19-BEJ1120 


\title{
Random walk approximation of BSDEs with Hölder continuous terminal condition
}

\author{
CHRISTEL GEISS ${ }^{1, *}$, CÉLINE LABART ${ }^{2}$ and ANTTI LUOTO ${ }^{1, * *}$ \\ ${ }^{1}$ Department of Mathematics and Statistics, University of Jyvaskyla, P.O. Box 35 (MaD), FI-40014, Fin- \\ land.E-mail: *christel.geiss@jyu.fi; **antti.k.luoto@jyu.fi \\ ${ }^{2}$ Univ. Grenoble Alpes, Univ. Savoie Mont Blanc, CNRS, LAMA, 73000 Chambéry, France. \\ E-mail: celine.labart@univ-smb.fr
}

In this paper, we consider the random walk approximation of the solution of a Markovian BSDE whose terminal condition is a locally Hölder continuous function of the Brownian motion. We state the rate of the $L_{2}$-convergence of the approximated solution to the true one. The proof relies in part on growth and smoothness properties of the solution $u$ of the associated PDE. Here we improve existing results by showing some properties of the second derivative of $u$ in space.

Keywords: backward stochastic differential equations; numerical scheme; random walk approximation; speed of convergence

\section{Introduction}

Let $(\Omega, \mathcal{F}, \mathbb{P})$ be a complete probability space carrying the standard Brownian motion $B=$ $\left(B_{t}\right)_{t \geq 0}$ and assume $\left(\mathcal{F}_{t}\right)_{t \geq 0}$ is the augmented natural filtration. We consider the following backward stochastic differential equation (BSDE for short)

$$
Y_{s}=g\left(B_{T}\right)+\int_{s}^{T} f\left(r, B_{r}, Y_{r}, Z_{r}\right) d r-\int_{s}^{T} Z_{r} d B_{r}, \quad 0 \leq s \leq T,
$$

where $f$ is Lipschitz continuous and $g$ is a locally $\alpha$-Hölder continuous and polynomially bounded function (see (3)). In this paper, we are interested in the $L_{2}$-convergence of the numerical approximation of (1) by using a random walk. First, results dealing with the numerical approximation of BSDEs date back to the late 1990s. Bally (see [2]) was the first to consider this problem by introducing random discretization, namely the jump times of a Poisson process. In his Ph.D. thesis, Chevance (see [17]) proposed the following discretization

$$
y_{k}=\mathbb{E}\left(y_{k+1}+h f\left(y_{k+1}\right) \mid \mathcal{F}_{k}^{n}\right), \quad k=n-1, \ldots, 0, n \in \mathbb{N}^{*}
$$

and proved the convergence of $\left(Y_{t}^{n}\right)_{t}:=\left(y_{[t / h]}\right)_{t}$ to $Y$. At the same time, Coquet, Mackevičius and Mémin [18] proved the convergence of $Y^{n}$ by using convergence of filtrations, still in the case of a generator independent from $z$. The general case ( $f$ depends on $z$, terminal condition $\xi \in L_{2}$ ) has been studied by Briand, Delyon and Mémin (see [7]). In that paper the authors define an approximated solution $\left(Y^{n}, Z^{n}\right)$ based on random walk and prove weak convergence to 
$(Y, Z)$ using convergence of filtrations. We also refer to [27,29-31] for other numerical methods for BSDEs which use a random walk approach. The rate of convergence of this method was left as an open problem.

Introducing instead of random walk an approach based on the dynamic programming equation, Bouchard and Touzi in [6] and Zhang in [36] managed to establish a rate of convergence. However, to be fully implementable, this algorithm requires to have a good approximation of its associated conditional expectation. For this, various methods have been developed (see [13, 20,25]). Forward methods have also been introduced to approximate (1): a branching diffusion method (see [26]), a multilevel Picard approximation (see [34]) and Wiener chaos expansion (see [9]). Many extensions of (1) have also been considered: high order schemes (see [10,11]), schemes for reflected BSDEs (see [3,15]), for fully-coupled BSDEs (see [4,21]), for quadratic BSDEs (see [14]), for BSDEs with jumps (see [23]) and for McKean-Vlasov BSDEs (see [1,12, 16]).

From a numerical point of view, the random walk is of course not competitive with recent methods listed above. We emphasize that the aim of this paper is to give the convergence rate of the initial method based on random walk, which, to the best of our knowledge, has not been done so far.

As in [7], let us introduce the following approximation of $B$, based on a random walk:

$$
B_{t}^{n}=\sqrt{h} \sum_{i=1}^{[t / h]} \varepsilon_{i}, \quad 0 \leq t \leq T
$$

where $h=\frac{T}{n}\left(n \in \mathbb{N}^{*}\right)$ and $\left(\varepsilon_{i}\right)_{i=1,2, \ldots}$ is a sequence of i.i.d. Rademacher random variables. Consider the following approximated solution $\left(Y^{n}, Z^{n}\right)$ of $(Y, Z)$

$$
Y_{t_{k}}^{n}=g\left(B_{T}^{n}\right)+h \sum_{m=k}^{n-1} f\left(t_{m+1}, B_{t_{m}}^{n}, Y_{t_{m}}^{n}, Z_{t_{m}}^{n}\right)-\sqrt{h} \sum_{m=k}^{n-1} Z_{t_{m}}^{n} \varepsilon_{m+1}, \quad 0 \leq k \leq n-1 .
$$

The main result of our paper gives the rate of convergence in $L_{2}$-norm of $Y_{v}^{n}-Y_{v}$ and $Z_{v}^{n}-Z_{v}$ for each $v \in\left[0, T\right.$ ) (see Theorem 3.1). Basically, we get that the $L_{2}$-norm of the error on $Y$ is of order $h^{\frac{\alpha}{4}}$ and the $L_{2}$-norm of the error on $Z$ is of order $\frac{h^{\frac{\alpha}{4}}}{\sqrt{T-v}}$. The proof of this result is based on several ingredients. In particular, we need some estimates on the bound of the first and second derivatives of the solution of the PDE associated to the BSDE (1). We establish these bounds in the case of a forward backward SDE (FBSDE for short) whose terminal condition satisfies the Hölder continuity condition (3). This result extends Zhang [37], Theorem 3.2.

The rest of the paper is organized as follows. Section 2 introduces notations, assumptions and the representation for $Z$ and $Z^{n}$ based on the Malliavin weights. Section 3 states the rate of convergence of the error on $Y$ and $Z$ in $L_{2}$-norm, which is the main result of the paper. Section 4 presents numerical simulations and Section 5 recalls some properties of Malliavin weights, of the regularity of solutions to FBSDEs with a locally Hölder continuous terminal condition function and states some properties of the solutions to the PDEs associated to these FBSDEs. 


\section{Preliminaries}

This section is dedicated to notations, assumptions and the representation of $Z$ and $Z^{n}$ using the Malliavin weights.

\section{Notation.}

- $\mathcal{G}_{k}:=\sigma\left(\varepsilon_{i}: 1 \leq i \leq k\right)$ and $\mathcal{G}_{0}=\{\varnothing, \Omega\}$. The associated discrete-time random walk $\left(B_{t_{k}}^{n}\right)_{k=0}^{n}$ is $\left(\mathcal{G}_{k}\right)_{k=0}^{n}$-adapted.

- $\|\cdot\|_{p}:=\|\cdot\|_{L^{p}(\mathbb{P})}$ for $p \geq 1$ and for $p=2$ simply $\|\cdot\|$.

\section{Assumption 2.1.}

- $g$ is locally Hölder continuous with order $\alpha \in(0,1]$ and polynomially bounded $\left(p_{0} \geq 0\right.$, $C_{g}>0$ ) in the following sense

$$
\forall(x, y) \in \mathbb{R}^{2}, \quad|g(x)-g(y)| \leq C_{g}\left(1+|x|^{p_{0}}+|y|^{p_{0}}\right)|x-y|^{\alpha} .
$$

- The function $[0, T] \times \mathbb{R}^{3}:(t, x, y, z) \mapsto f(t, x, y, z)$ satisfies

$$
\left|f(t, x, y, z)-f\left(t^{\prime}, x^{\prime}, y^{\prime}, z^{\prime}\right)\right| \leq L_{f}\left(\sqrt{t-t^{\prime}}+\left|x-x^{\prime}\right|+\left|y-y^{\prime}\right|+\left|z-z^{\prime}\right|\right) .
$$

Notice that (3) implies

$$
|g(x)| \leq K\left(1+|x|^{p_{0}+1}\right)=: \Psi(x) \text {. }
$$

In the rest of the paper, the study of the error $\left(Y^{n}-Y, Z^{n}-Z\right)$ will either rely on (2) or on its integral version:

$$
Y_{s}^{n}=g\left(B_{T}^{n}\right)+\int_{(s, T]} f\left(r, B_{r^{-}}^{n}, Y_{r^{-}}^{n}, Z_{r^{-}}^{n}\right) d\left[B^{n}, B^{n}\right]_{r}-\int_{(s, T]} Z_{r^{-}}^{n} d B_{r}^{n}, \quad 0 \leq s \leq T,
$$

where the backward equation (6) arises from (2) by setting $Y_{r}^{n}:=Y_{t_{m}}^{n}$ and $Z_{r}^{n}:=Z_{t_{m}}^{n}$ for $r \in$ $\left[t_{m}, t_{m+1}\right)$. For $n$ large enough, (6) has a unique solution $\left(Y^{n}, Z^{n}\right)$, and $\left(Y_{t_{m}}^{n}, Z_{t_{m}}^{n}\right)_{m=0}^{n-1}$ is adapted to the filtration $\left(\mathcal{G}_{m}\right)_{m=0}^{n-1}$. Let us now introduce the Malliavin representations for $Z$ and $Z^{n}$. They are the cornerstone of our study of the error on $Z$.

\subsection{Representations for $Z$ and $Z^{n}$}

We will use the representation (see Ma and Zhang [28], Theorem 4.2)

$$
Z_{t}=\mathbb{E}_{t}\left(g\left(B_{T}\right) N_{T}^{t}+\int_{t}^{T} f\left(s, B_{s}, Y_{s}, Z_{s}\right) N_{s}^{t} d s\right), \quad 0 \leq t \leq T,
$$

where $\mathbb{E}_{t}[\cdot]=\mathbb{E}\left[\cdot \mid \mathcal{F}_{t}\right]$, and for all $s \in(t, T]$ we have

$$
N_{s}^{t}:=\frac{B_{s}-B_{t}}{s-t}
$$


Lemma 2.2. Suppose that Assumption 2.1 holds. Then the process $Z^{n}$ given by (6) has the representation

$$
Z_{t_{k}}^{n}=\mathbb{E}_{k}\left(g\left(B_{T}^{n}\right) \frac{B_{t_{n}}^{n}-B_{t_{k}}^{n}}{t_{n}-t_{k}}\right)+\mathbb{E}_{k}\left(h \sum_{m=k+1}^{n-1} f\left(t_{m+1}, B_{t_{m}}^{n}, Y_{t_{m}}^{n}, Z_{t_{m}}^{n}\right) \frac{B_{t_{m}}^{n}-B_{t_{k}}^{n}}{t_{m}-t_{k}}\right)
$$

for $k=0,1, \ldots, n-1$, where $\mathbb{E}_{k}[\cdot]:=\mathbb{E}\left[\cdot \mid \mathcal{G}_{k}\right]$.

Proof. We multiply equation (2) by $\varepsilon_{k+1}$ and take the conditional expectation with respect to $\mathcal{G}_{k}$. Since $\left(Y_{t_{k}}^{n}, Z_{t_{k}}^{n}\right)$ is $\mathcal{G}_{k}$-measurable, it holds for $0 \leq k \leq n-1$ that

$$
\begin{aligned}
\mathbb{E}_{k}\left(Y_{t_{k}}^{n} \varepsilon_{k+1}\right) & \\
= & \mathbb{E}_{k}\left(g\left(B_{T}^{n}\right) \varepsilon_{k+1}\right)+h \mathbb{E}_{k}\left(\sum_{m=k}^{n-1} f\left(t_{m+1}, B_{t_{m}}^{n}, Y_{t_{m}}^{n}, Z_{t_{m}}^{n}\right) \varepsilon_{k+1}\right)-\sqrt{h} \mathbb{E}_{k}\left(\sum_{m=k}^{n-1} Z_{t_{m}}^{n} \varepsilon_{m+1} \varepsilon_{k+1}\right) \\
= & \sqrt{h} \mathbb{E}_{k}\left(g\left(B_{T}^{n}\right) \frac{B_{t_{n}}^{n}-B_{t_{k}}^{n}}{t_{n}-t_{k}}\right)+h^{3 / 2} \sum_{m=k+1}^{n-1} \mathbb{E}_{k}\left(f\left(t_{m+1}, B_{t_{m}}^{n}, Y_{t_{m}}^{n}, Z_{t_{m}}^{n}\right) \frac{B_{t_{m}}^{n}-B_{t_{k}}^{n}}{t_{m}-t_{k}}\right) \\
& -\sqrt{h} Z_{t_{k}}^{n},
\end{aligned}
$$

where the 1.h.s. is equal to zero. Indeed, for $m \geq k+1$, we have

$$
\mathbb{E}_{k}\left(Z_{t_{m}}^{n} \varepsilon_{m+1} \varepsilon_{k+1} h\right)=\mathbb{E}_{k}\left(Z_{t_{m}}^{n} \varepsilon_{k+1} \mathbb{E}_{m} \varepsilon_{m+1}\right)=0
$$

and for $m=k$ it holds $\mathbb{E}_{k}\left(Z_{t_{k}}^{n} \varepsilon_{k+1}^{2}\right)=Z_{t_{k}}^{n}$. Moreover, the fact that $B_{T}^{n}=\sqrt{h} \sum_{m=0}^{n-1} \varepsilon_{m+1}$, where $\left(\varepsilon_{m}\right)_{m=1,2 \ldots}$ are i.i.d., yields

$$
\begin{aligned}
\mathbb{E}_{k}\left(g\left(B_{T}^{n}\right) \varepsilon_{k+1}\right) & =\mathbb{E}_{k}\left(g\left(B_{T}^{n}\right) \sum_{m=k}^{n-1} \frac{\varepsilon_{k+1}}{n-k}\right)=\mathbb{E}_{k}\left(g\left(B_{T}^{n}\right) \sum_{m=k}^{n-1} \frac{\varepsilon_{m+1}}{n-k}\right) \\
& =\sqrt{h} \mathbb{E}_{k}\left(g\left(B_{T}^{n}\right) \frac{B_{t_{n}}^{n}-B_{t_{k}}^{n}}{t_{n}-t_{k}}\right) .
\end{aligned}
$$

Similarly, for $m \geq k+1$, we get (using [7], Proposition 5.1, where it is stated that both $Y_{t_{m}}^{n}$ and $Z_{t_{m}}^{n}$ can be represented as functions of $t_{m}$ and $B_{t_{m}}^{n}$ )

$$
\mathbb{E}_{k}\left(f\left(t_{m+1}, B_{t_{m}}^{n}, Y_{t_{m}}^{n}, Z_{t_{m}}^{n}\right) \varepsilon_{k+1}\right)=\sqrt{h} \mathbb{E}_{k}\left(f\left(t_{m+1}, B_{t_{m}}^{n}, Y_{t_{m}}^{n}, Z_{t_{m}}^{n}\right) \frac{B_{t_{m}}^{n}-B_{t_{k}}^{n}}{t_{m}-t_{k}}\right)
$$

It remains to divide (9) by $\sqrt{h}$ and rearrange. 


\section{Main result}

This section is devoted to the main result of the paper: the rate of the $L_{2}$-convergence of $\left(Y^{n}, Z^{n}\right)$ to $(Y, Z)$. The proof will rely on the fact that the random walk $B^{n}$ can be constructed from the Brownian motion $B$ by Skorohod embedding. Let $\tau_{0}:=0$ and define

$$
\tau_{k}:=\inf \left\{t>\tau_{k-1}:\left|B_{t}-B_{\tau_{k-1}}\right|=\sqrt{h}\right\}, \quad k \geq 1 .
$$

Then $\left(B_{\tau_{k}}-B_{\tau_{k-1}}\right)_{k=1}^{\infty}$ is a sequence of i.i.d. random variables with

$$
\mathbb{P}\left(B_{\tau_{k}}-B_{\tau_{k-1}}= \pm \sqrt{h}\right)=\frac{1}{2}
$$

which means that $\sqrt{h} \varepsilon_{k} \stackrel{d}{=} B_{\tau_{k}}-B_{\tau_{k-1}}$. We will use this random walk for our approximation, that is, we will require

$$
B_{t}^{n}=\sum_{k=1}^{[t / h]}\left(B_{\tau_{k}}-B_{\tau_{k-1}}\right), \quad 0 \leq t \leq T .
$$

Properties satisfied by $\tau_{k}$ and $B_{\tau_{k}}$ are stated in Lemma A.1. We will denote by $\mathbb{E}_{\tau_{k}}$ the conditional expectation w.r.t. $\mathcal{F}_{\tau_{k}}$.

Theorem 3.1. Let Assumption 2.1 hold. If $B^{n}$ satisfies (10) then we have (for sufficiently large $n$ ) that

$$
\begin{aligned}
& \mathbb{E}\left|Y_{v}-Y_{v}^{n}\right|^{2} \leq C_{0} h^{\frac{\alpha}{2}} \quad \text { for } v \in[0, T), \\
& \mathbb{E}\left|Z_{v}-Z_{v}^{n}\right|^{2} \leq C_{0} \frac{h^{\frac{\alpha}{2}}}{T-t_{k}}+C_{1} \frac{h^{\frac{\alpha}{2}}}{(T-v)^{1-\frac{\alpha}{2}}} \mathbf{1}_{v \neq t_{k}} \quad \text { for } v \in\left[t_{k}, t_{k+1}\right), k=0, \ldots, n-1,
\end{aligned}
$$

where we have the dependencies $C_{0}=C\left(T, p_{0}, L_{f}, C_{g}, C_{5.3}^{y}, C_{5.3}^{z}, K_{f}, c_{5.4}, \alpha\right), C_{1}=C\left(T, p_{0}\right.$, $\left.C_{5.3}^{z}, \alpha\right)$ and $K_{f}:=\sup _{0 \leq t \leq T}|f(t, 0,0,0)|$.

Remark 3.2. Theorem 3.1 implies that

$$
\sup _{v \in[0, T)} \mathbb{E}\left|Y_{v}-Y_{v}^{n}\right|^{2} \leq C_{0} h^{\frac{\alpha}{2}} \quad \text { and } \quad \mathbb{E} \int_{0}^{T}\left|Z_{v}-Z_{v}^{n}\right|^{2} d v \leq C\left(C_{0}, C_{1}, \beta\right) h^{\beta} \quad \text { for } \beta \in\left(0, \frac{\alpha}{2}\right) \text {. }
$$

Proof of Theorem 3.1. Let $u:[0, T) \times \mathbb{R} \rightarrow \mathbb{R}$ be the solution of the PDE associated to (1). Since by Theorem 5.4

$$
Y_{s}=u\left(s, B_{s}\right), \quad Z_{s}=u_{x}\left(s, B_{s}\right), \quad \text { a.s. }
$$

we introduce

$$
F(s, x):=f\left(s, x, u(s, x), u_{x}(s, x)\right),
$$

so that $F\left(s, B_{s}\right)=f\left(s, B_{s}, Y_{s}, Z_{s}\right)$. We first give some properties satisfied by $F$. 
Lemma 3.3. If Assumption 2.1 holds then $F$ is a Lipschitz continuous and polynomially bounded function in $x$ :

$$
\begin{gathered}
\left|F\left(t, x_{1}\right)-F\left(t, x_{2}\right)\right| \leq C\left(T, L_{f}, c_{5.4}^{2,3}\right)\left(1+\left|x_{1}\right|^{p_{0}+1}+\left|x_{2}\right|^{p_{0}+1}\right) \frac{\left|x_{1}-x_{2}\right|}{(T-t)^{1-\frac{\alpha}{2}}}, \\
|F(t, x)| \leq C\left(T, L_{f}, c_{5.4}^{1,2}, K_{f}\right) \frac{\Psi(x)}{(T-t)^{\frac{1-\alpha}{2}}}
\end{gathered}
$$

where $\Psi(x)$ is given in (5).

Proof of Lemma 3.3. Thanks to the mean value theorem and Theorem 5.4-(ii-c) and (iii-b) we have for $x_{1}, x_{2} \in \mathbb{R}$ that there exist $\xi_{1}, \xi_{2} \in\left[\min \left\{x_{1}, x_{2}\right\}, \max \left\{x_{1}, x_{2}\right\}\right]$ such that

$$
\begin{aligned}
\left|F\left(t, x_{1}\right)-F\left(t, x_{2}\right)\right| & =\left|f\left(t, x_{1}, u\left(t, x_{1}\right), u_{x}\left(t, x_{1}\right)\right)-f\left(t, x_{2}, u\left(t, x_{2}\right), u_{x}\left(t, x_{2}\right)\right)\right| \\
& \leq L_{f}\left(\left|x_{1}-x_{2}\right|+\left|u\left(t, x_{1}\right)-u\left(t, x_{2}\right)\right|+\left|u_{x}\left(t, x_{1}\right)-u_{x}\left(t, x_{2}\right)\right|\right) \\
& \leq L_{f}\left(1+\frac{c_{5.4}^{2} \Psi\left(\xi_{1}\right)}{(T-t)^{\frac{1-\alpha}{2}}}+\frac{c_{5.4}^{3} \Psi\left(\xi_{2}\right)}{(T-t)^{1-\frac{\alpha}{2}}}\right)\left|x_{1}-x_{2}\right| \\
& \leq C\left(T, L_{f}, c_{5.4}^{2,3}\right)\left(1+\left|x_{1}\right|^{p_{0}+1}+\left|x_{2}\right|^{p_{0}+1}\right) \frac{\left|x_{1}-x_{2}\right|}{(T-t)^{1-\frac{\alpha}{2}}} .
\end{aligned}
$$

The second inequality can be shown similarly.

For the estimate of $\mathbb{E}\left|Y_{t_{k}}-Y_{t_{k}}^{n}\right|^{2}$ we will use (1) and (2): Since $Y_{t_{k}}^{n}$ is $\mathcal{F}_{\tau_{k}}$-measurable we have

$$
\begin{aligned}
\left\|Y_{t_{k}}-Y_{t_{k}}^{n}\right\| \leq & \left\|\mathbb{E}_{t_{k}} g\left(B_{T}\right)-\mathbb{E}_{\tau_{k}} g\left(B_{T}^{n}\right)\right\| \\
& +\left\|\mathbb{E}_{t_{k}} \int_{t_{k}}^{T} f\left(s, B_{s}, Y_{s}, Z_{s}\right) d s-h \mathbb{E}_{\tau_{k}} \sum_{m=k}^{n-1} f\left(t_{m+1}, B_{t_{m}}^{n}, Y_{t_{m}}^{n}, Z_{t_{m}}^{n}\right)\right\| .
\end{aligned}
$$

We frequently express conditional expectations with the help of an independent copy of $B$ denoted by $\tilde{B}$, for example, $\mathbb{E}_{t} g\left(B_{T}\right)=\tilde{\mathbb{E}} g\left(B_{t}+\tilde{B}_{T-t}\right)$.

By (3) and Lemma A.1,

$$
\begin{aligned}
\left\|\mathbb{E}_{t_{k}} g\left(B_{T}\right)-\mathbb{E}_{\tau_{k}} g\left(B_{T}^{n}\right)\right\|^{2} & =\mathbb{E}\left|\tilde{\mathbb{E}} g\left(B_{t_{k}}+\tilde{B}_{T-t_{k}}\right)-\tilde{\mathbb{E}} g\left(B_{\tau_{k}}+\tilde{B}_{\tilde{\tau}_{n-k}}\right)\right|^{2} \\
& \leq\left(\mathbb{E} \tilde{\mathbb{E}}\left(\Psi_{1}\right)^{4}\right)^{\frac{1}{2}}\left(\mathbb{E} \tilde{\mathbb{E}}\left|B_{t_{k}}-B_{\tau_{k}}+\tilde{B}_{T-t_{k}}-\tilde{B}_{\tilde{\tau}_{n-k}}\right|^{4 \alpha}\right)^{\frac{1}{2}} \\
& \leq C\left(C_{g}, T, p_{0}\right)\left(\left(\mathbb{E}\left|B_{t_{k}}-B_{\tau_{k}}\right|^{4 \alpha}\right)^{\frac{1}{2}}+\left(\mathbb{E}\left|B_{T-t_{k}}-B_{\tau_{n-k}}\right|^{4 \alpha}\right)^{\frac{1}{2}}\right) \\
& \leq C\left(C_{g}, T, p_{0}\right) h^{\frac{\alpha}{2}}
\end{aligned}
$$


where $\Psi_{1}:=C_{g}\left(1+\left|B_{t_{k}}+\tilde{B}_{T-t_{k}}\right|^{p_{0}}+\left|B_{\tau_{k}}+\tilde{B}_{\tilde{\tau}_{n-k}}\right|^{p_{0}}\right)$. To estimate the other term in (11), we consider the decomposition

$$
\begin{aligned}
\mathbb{E}_{t_{k}} f\left(s, B_{s}, Y_{s}, Z_{s}\right)-\mathbb{E}_{\tau_{k}} f\left(t_{m+1}, B_{t_{m}}^{n}, Y_{t_{m}}^{n}, Z_{t_{m}}^{n}\right) \\
=\left(\mathbb{E}_{t_{k}} f\left(s, B_{s}, Y_{s}, Z_{s}\right)-\mathbb{E}_{t_{k}} f\left(t_{m}, B_{t_{m}}, Y_{t_{m}}, Z_{t_{m}}\right)\right)+\left(\mathbb{E}_{t_{k}} F\left(t_{m}, B_{t_{m}}\right)-\mathbb{E}_{\tau_{k}} F\left(t_{m}, B_{\tau_{m}}\right)\right) \\
\quad+\left(\mathbb{E}_{\tau_{k}} F\left(t_{m}, B_{\tau_{m}}\right)-\mathbb{E}_{\tau_{k}} F\left(t_{m}, B_{t_{m}}\right)\right) \\
\quad+\left(\mathbb{E}_{\tau_{k}} f\left(t_{m}, B_{t_{m}}, Y_{t_{m}}, Z_{t_{m}}\right)-\mathbb{E}_{\tau_{k}} f\left(t_{m+1}, B_{t_{m}}^{n}, Y_{t_{m}}^{n}, Z_{t_{m}}^{n}\right)\right) \\
=: D_{1}(s, m)+D_{2}(m)+\cdots+D_{4}(m)
\end{aligned}
$$

so that

$$
\begin{aligned}
& \left\|\mathbb{E}_{t_{k}} \int_{t_{k}}^{T} f\left(s, B_{s}, Y_{s}, Z_{s}\right) d s-h \mathbb{E}_{\tau_{k}} \sum_{m=k}^{n-1} f\left(t_{m+1}, B_{t_{m}}^{n}, Y_{t_{m}}^{n}, Z_{t_{m}}^{n}\right)\right\| \\
& \quad \leq \sum_{m=k}^{n-1}\left(\left\|\int_{t_{m}}^{t_{m+1}} D_{1}(s, m) d s\right\|+h \sum_{i=2}^{4}\left\|D_{i}(m)\right\|\right) .
\end{aligned}
$$

For $D_{1}$ we have by Theorem 5.3 that

$$
\begin{aligned}
\left\|D_{1}(s, m)\right\| & \leq L_{f}\left(\sqrt{s-t_{m}}+\left\|B_{s}-B_{t_{m}}\right\|+\left\|Y_{s}-Y_{t_{m}}\right\|+\left\|Z_{s}-Z_{t_{m}}\right\|\right) \\
& \leq C\left(T, L_{f}, C_{5.3}^{y}, C_{5.3}^{z}, p_{0}\right)(T-s)^{\frac{\alpha-2}{2}} h^{\frac{1}{2}},
\end{aligned}
$$

where the last inequality follows from $\left\|B_{s}-B_{t_{m}}\right\|=\sqrt{s-t_{m}} \leq h^{\frac{1}{2}}$ for $s \in\left[t_{m}, t_{m+1}\right]$ and

$$
\begin{aligned}
\| Y_{s} & -Y_{t_{m}}\|+\| Z_{s}-Z_{t_{m}} \| \\
& \leq\left(\mathbb{E} \Psi\left(B_{t_{m}}\right)^{2}\right)^{\frac{1}{2}}\left(C_{5.3}^{y}\left(\int_{t_{m}}^{s}(T-r)^{\alpha-1} d r\right)^{\frac{1}{2}}+C_{5.3}^{z}\left(\int_{t_{m}}^{s}(T-r)^{\alpha-2} d r\right)^{\frac{1}{2}}\right) \\
& \leq C\left(T, C_{5.3}^{y}, C_{5.3}^{z}, p_{0}\right) \sqrt{s-t_{m}}\left((T-s)^{\frac{\alpha-1}{2}}+(T-s)^{\frac{\alpha-2}{2}}\right) .
\end{aligned}
$$

We bound $D_{2}$ using Lemma 3.3 and Lemma A.1. Similar to (12) we conclude (setting $\Psi_{2}:=$ $\left.1+\left|B_{t_{k}}+\tilde{B}_{t_{m-k}}\right|^{p_{0}+1}+\left|B_{\tau_{k}}+\tilde{B}_{\tilde{\tau}_{m-k}}\right|^{p_{0}+1}\right)$ that

$$
\begin{aligned}
\left\|D_{2}(m)\right\| & =\left(\mathbb{E}\left|\mathbb{E}_{t_{k}} F\left(t_{m}, B_{t_{m}}\right)-\mathbb{E}_{\tau_{k}} F\left(t_{m}, B_{\tau_{m}}\right)\right|^{2}\right)^{\frac{1}{2}} \\
& \leq C\left(T, L_{f}, c_{5.4}^{2,3}\right)\left(\mathbb{E} \tilde{\mathbb{E}} \Psi_{2}^{4}\right)^{\frac{1}{4}} \frac{1}{\left(T-t_{m}\right)^{1-\frac{\alpha}{2}}}\left(t_{k} h+t_{m-k} h\right)^{\frac{1}{4}} \\
& \leq C\left(T, p_{0}, L_{f}, c_{5.4}^{2,3}\right) \frac{1}{\left(T-t_{m}\right)^{1-\frac{\alpha}{2}}} h^{\frac{1}{4}} .
\end{aligned}
$$


For $D_{3}$ we apply again Lemma 3.3 and Lemma A.1,

$$
\begin{aligned}
\left\|D_{3}(m)\right\| & \leq\left\|F\left(t_{m}, B_{t_{m}}\right)-F\left(t_{m}, B_{\tau_{m}}\right)\right\| \leq C\left(T, L_{f}, c_{5.4}^{2,3}\right) \frac{1}{\left(T-t_{m}\right)^{1-\frac{\alpha}{2}}}\left\|\Psi_{3}\left|B_{t_{m}}-B_{\tau_{m}}\right|\right\| \\
& \leq C\left(T, p_{0}, L_{f}, c_{5.4}^{2,3}\right) \frac{1}{\left(T-t_{m}\right)^{1-\frac{\alpha}{2}}} h^{\frac{1}{4}}
\end{aligned}
$$

where $\Psi_{3}:=1+\left|B_{t_{m}}\right|^{p_{0}+1}+\left|B_{\tau_{m}}\right|^{p_{0}+1}$. For the last term $D_{4}$ we get

$$
\left\|D_{4}(m)\right\| \leq L_{f}\left(h^{\frac{1}{2}}+\left\|B_{t_{m}}-B_{t_{m}}^{n}\right\|+\left\|Y_{t_{m}}-Y_{t_{m}}^{n}\right\|+\left\|Z_{t_{m}}-Z_{t_{m}}^{n}\right\|\right) .
$$

Finally, using the estimates for the terms $D_{1}(s, m), D_{2}(m), \ldots, D_{4}(m)$ we arrive at

$$
\begin{aligned}
\left\|Y_{t_{k}}-Y_{t_{k}}^{n}\right\| \leq & C\left(C_{g}, T, p_{0}\right) h^{\frac{\alpha}{4}}+C\left(T, L_{f}, C_{5.3}^{y}, C_{5.3}^{z}, p_{0}\right) h^{\frac{1}{2}} \int_{t_{k}}^{T}(T-s)^{\frac{\alpha-2}{2}} d s \\
& +C\left(T, p_{0}, L_{f}, c_{5.4}^{2,3}\right) h^{\frac{1}{4}} \sum_{m=k}^{n-1} \frac{h}{\left(T-t_{m}\right)^{1-\frac{\alpha}{2}}} \\
& +h L_{f} \sum_{m=k}^{n-1}\left(\left\|Y_{t_{m}}-Y_{t_{m}}^{n}\right\|+\left\|Z_{t_{m}}-Z_{t_{m}}^{n}\right\|\right) \\
\leq & C\left(C_{g}, T, p_{0}, L_{f}, c_{5.4}^{2,3}, C_{5.3}^{y}, C_{5.3}^{z}\right)^{\frac{\alpha}{4}} \\
& +h L_{f} \sum_{m=k}^{n-1}\left(\left\|Y_{t_{m}}-Y_{t_{m}}^{n}\right\|+\left\|Z_{t_{m}}-Z_{t_{m}}^{n}\right\|\right) .
\end{aligned}
$$

For $\left\|Z_{t_{k}}-Z_{t_{k}}^{n}\right\|$ we exploit the representations (7) and (8) and estimate

$$
\begin{aligned}
\left\|Z_{t_{k}}-Z_{t_{k}}^{n}\right\| \leq & \frac{1}{T-t_{k}}\left\|\mathbb{E}_{t_{k}} g\left(B_{T}\right)\left(B_{T}-B_{t_{k}}\right)-\mathbb{E}_{\tau_{k}} g\left(B_{\tau_{n}}\right)\left(B_{\tau_{n}}-B_{\tau_{k}}\right)\right\| \\
& +\| \mathbb{E}_{t_{k}}\left(\int_{t_{k+1}}^{T} f\left(s, B_{s}, Y_{s}, Z_{s}\right) \frac{B_{s}-B_{t_{k}}}{s-t_{k}} d s\right) \\
& -\mathbb{E}_{\tau_{k}}\left(h \sum_{m=k+1}^{n-1} f\left(t_{m+1}, B_{t_{m}}^{n}, Y_{t_{m}}^{n}, Z_{t_{m}}^{n}\right) \frac{B_{t_{m}}^{n}-B_{t_{k}}^{n}}{t_{m}-t_{k}}\right) \| \\
& +\left\|\mathbb{E}_{t_{k}} \int_{t_{k}}^{t_{k+1}} f\left(s, B_{s}, Y_{s}, Z_{s}\right) \frac{B_{s}-B_{t_{k}}}{s-t_{k}} d s\right\| .
\end{aligned}
$$

Then, similar to (12), we have for the terminal condition by Lemma A.1 that

$$
\begin{array}{r}
\left\|\mathbb{E}_{t_{k}}\left[g\left(B_{T}\right)\left(B_{T}-B_{t_{k}}\right)\right]-\mathbb{E}_{\tau_{k}}\left[g\left(B_{\tau_{n}}\right)\left(B_{\tau_{n}}-B_{\tau_{k}}\right)\right]\right\| \\
=\| \tilde{\mathbb{E}}\left[g\left(B_{t_{k}}+\tilde{B}_{T-t_{k}}\right)-g\left(B_{t_{k}}\right)\right]\left(\tilde{B}_{T-t_{k}}-\tilde{B}_{\tilde{\tau}_{n-k}}\right)
\end{array}
$$




$$
\begin{aligned}
& +\tilde{\mathbb{E}}\left[g\left(B_{t_{k}}+\tilde{B}_{T-t_{k}}\right)-g\left(B_{\tau_{k}}+\tilde{B}_{\tilde{\tau}_{n-k}}\right)\right] \tilde{B}_{\tilde{\tau}_{n-k}} \| \\
\leq & C\left(C_{g}, T, p_{0}\right) h^{\frac{1}{4}}\left(T-t_{k}\right)^{\frac{\alpha}{2}+\frac{1}{4}}+C\left(C_{g}, T, p_{0}\right) h^{\frac{\alpha}{4}}\left(T-t_{k}\right)^{\frac{1}{2}} \leq C\left(C_{g}, T, p_{0}\right) h^{\frac{\alpha}{4}}\left(T-t_{k}\right)^{\frac{1}{2}} .
\end{aligned}
$$

Here we have used that $\tilde{\mathbb{E}}\left[g\left(B_{t_{k}}\right)\left(\tilde{B}_{T-t_{k}}-\tilde{B}_{\tilde{\tau}_{n-k}}\right)\right]=0$. The term $\tilde{\mathbb{E}}\left[g\left(B_{t_{k}}+\tilde{B}_{T-t_{k}}\right)-\right.$ $\left.g\left(B_{t_{k}}\right)\right]\left(\tilde{B}_{T-t_{k}}-\tilde{B}_{\tilde{\tau}_{n-k}}\right)$ provides us with the factor $\left(T-t_{k}\right)^{\frac{\alpha}{2}}\left(\left(T-t_{k}\right) h\right)^{\frac{1}{4}}$. For the next term of the estimate of $\left\|Z_{t_{k}}-Z_{t_{k}}^{n}\right\|$ we use for $s \in\left[t_{m}, t_{m+1}\right)$, where $m \geq k+1$, the decomposition

$$
\begin{aligned}
& \frac{\mathbb{E}_{t_{k}} f\left(s, B_{s}, Y_{s}, Z_{s}\right)\left(B_{s}-B_{t_{k}}\right)}{s-t_{k}}-\frac{\mathbb{E}_{\tau_{k}} f\left(t_{m+1}, B_{t_{m}}^{n}, Y_{t_{m}}^{n}, Z_{t_{m}}^{n}\right)\left(B_{t_{m}}^{n}-B_{t_{k}}^{n}\right)}{t_{m}-t_{k}} \frac{\mathbb{E}_{t_{k}} f\left(s, B_{s}, Y_{s}, Z_{s}\right)\left(B_{s}-B_{t_{k}}\right)}{s-t_{k}}-\frac{\mathbb{E}_{t_{k}} f\left(t_{m}, B_{t_{m}}, Y_{t_{m}}, Z_{t_{m}}\right)\left(B_{t_{m}}-B_{t_{k}}\right)}{t_{m}-t_{k}} \\
& \quad+\frac{\mathbb{E}_{t_{k}} F\left(t_{m}, B_{t_{m}}\right)\left(B_{t_{m}}-B_{t_{k}}\right)}{t_{m}-t_{k}}-\frac{\mathbb{E}_{\tau_{k}} F\left(t_{m}, B_{\tau_{m}}\right)\left(B_{\tau_{m}}-B_{\tau_{k}}\right)}{t_{m}-t_{k}} \\
& \quad+\mathbb{E}_{\tau_{k}}\left[\left[F\left(t_{m}, B_{\tau_{m}}\right)-F\left(t_{m}, B_{t_{m}}\right)\right] \frac{B_{\tau_{m}}-B_{\tau_{k}}}{t_{m}-t_{k}}\right] \\
& \quad+\mathbb{E}_{\tau_{k}}\left[\left[f\left(t_{m}, B_{t_{m}}, Y_{t_{m}}, Z_{t_{m}}\right)-f\left(t_{m+1}, B_{t_{m}}^{n}, Y_{t_{m}}^{n}, Z_{t_{m}}^{n}\right)\right] \frac{B_{t_{m}}^{n}-B_{t_{k}}^{n}}{t_{m}-t_{k}}\right] \\
&=: T_{1}(s, m)+T_{2}(m)+\cdots+T_{4}(m) .
\end{aligned}
$$

Then by the conditional Hölder inequality and by (13) as well as by Lemma 3.3 we have

$$
\begin{aligned}
\left\|T_{1}(s, m)\right\| \leq & \left\|D_{1}(s, m)\right\| \frac{\left\|B_{s}-B_{t_{k}}\right\|}{s-t_{k}}+\left\|f\left(t_{m}, B_{t_{m}}, Y_{t_{m}}, Z_{t_{m}}\right)\right\|\left\|\frac{B_{s}-B_{t_{k}}}{s-t_{k}}-\frac{B_{t_{m}}-B_{t_{k}}}{t_{m}-t_{k}}\right\| \\
\leq & C\left(T, L_{f}, C_{5.3}^{y}, C_{5.3}^{z}, p_{0}\right)(T-s)^{\frac{\alpha-2}{2}} \frac{h^{\frac{1}{2}}}{\sqrt{s-t_{k}}} \\
& +C\left(T, L_{f}, c_{5.4}^{1,2}, K_{f}\right) \frac{\left(\mathbb{E} \Psi\left(B_{t_{m}}\right)^{2}\right)^{\frac{1}{2}}}{\left(T-t_{m}\right)^{\frac{1-\alpha}{2}}} \\
& \times\left(\frac{\left\|B_{s}-B_{t_{m}}\right\|}{s-t_{k}}+\left\|B_{t_{m}}-B_{t_{k}}\right\|\left|\frac{1}{s-t_{k}}-\frac{1}{t_{m}-t_{k}}\right|\right) \\
\leq & C\left(T, L_{f}, K_{f}, C_{5.3}^{y}, C_{5.3}^{z}, c_{5.4}^{1,2}, p_{0}\right)(T-s)^{\frac{\alpha-2}{2}} \frac{h^{\frac{1}{4}}}{\left(s-t_{k}\right)^{\frac{3}{4}}} .
\end{aligned}
$$

Indeed,

$$
\frac{\left\|B_{s}-B_{t_{m}}\right\|}{s-t_{k}}+\left\|B_{t_{m}}-B_{t_{k}}\right\|\left|\frac{1}{s-t_{k}}-\frac{1}{t_{m}-t_{k}}\right| \leq \frac{\sqrt{s-t_{m}}}{s-t_{k}}+\frac{\sqrt{t_{m}-t_{k}}\left(s-t_{m}\right)}{\left(s-t_{k}\right)\left(t_{m}-t_{k}\right)} \leq C \frac{h^{\frac{1}{4}}}{\left(s-t_{k}\right)^{\frac{3}{4}}},
$$


where the last inequality follows from $s-t_{m} \leq t_{m+1}-t_{m}=h$ and $h \leq t_{m}-t_{k} \leq s-t_{k}$. We estimate $T_{2}$ with the help of Lemma 3.3 and Lemma A.1 as follows:

$$
\begin{aligned}
\left\|T_{2}(m)\right\| & \leq\left\|\widehat{D}_{2}(m)\right\| \frac{\left\|B_{t_{m}}-B_{t_{k}}\right\|}{t_{m}-t_{k}}+\left\|F\left(t_{m}, B_{\tau_{m}}\right)\right\| \frac{\left\|B_{t_{m-k}}-B_{\tau_{m-k}}\right\|}{t_{m}-t_{k}} \\
& \leq C\left(T, p_{0}, L_{f}, K_{f}, c_{5.4}\right) \frac{1}{\left(T-t_{m}\right)^{1-\frac{\alpha}{2}}} \frac{h^{\frac{1}{4}}}{\left(t_{m}-t_{k}\right)^{\frac{3}{4}}} .
\end{aligned}
$$

Here $\widehat{D}_{2}(m):=\left(\tilde{\mathbb{E}}\left|F\left(t_{m}, B_{t_{k}}+\tilde{B}_{t_{m-k}}\right)-F\left(t_{m}, B_{\tau_{k}}+\tilde{B}_{\tilde{\tau}_{m-k}}\right)\right|^{2}\right)^{\frac{1}{2}}$ which can be estimated as $D_{2}(m)$. For $T_{3}$ the conditional Hölder inequality and Lemma A.1 yield

$$
\left\|T_{3}(m)\right\| \leq\left\|\widehat{D}_{3}(m)\right\|\left\|\frac{B_{\tau_{m}}-B_{\tau_{k}}}{t_{m}-t_{k}}\right\| \leq C\left(T, p_{0}, L_{f}, c_{5.4}^{2,3}\right) \frac{1}{\left(T-t_{m}\right)^{1-\frac{\alpha}{2}}} \frac{h^{\frac{1}{4}}}{\left(t_{m}-t_{k}\right)^{\frac{1}{2}}},
$$

where $\widehat{D}_{3}(m):=F\left(t_{m}, B_{\tau_{m}}\right)-F\left(t_{m}, B_{t_{m}}\right)$ is estimated as $D_{3}(m)$. Finally,

$$
\left\|T_{4}(m)\right\| \leq L_{f}\left(h^{\frac{1}{2}}+\left\|B_{t_{m}}-B_{t_{m}}^{n}\right\|+\left\|Y_{t_{m}}-Y_{t_{m}}^{n}\right\|+\left\|Z_{t_{m}}-Z_{t_{m}}^{n}\right\|\right) \frac{1}{\sqrt{t_{m}-t_{k}}} .
$$

For the estimate of $\left\|\mathbb{E}_{t_{k}} \int_{t_{k}}^{t_{k+1}} f\left(s, B_{s}, Y_{s}, Z_{s}\right) \frac{B_{s}-B_{t_{k}}}{s-t_{k}} d s\right\|$ one notices that by the conditional Hölder inequality,

$$
\begin{aligned}
\left\|\mathbb{E}_{t_{k}} f\left(s, B_{s}, Y_{s}, Z_{s}\right) \frac{B_{s}-B_{t_{k}}}{s-t_{k}}\right\| & =\left\|\mathbb{E}_{t_{k}}\left[\left(f\left(s, B_{s}, Y_{s}, Z_{s}\right)-f\left(s, B_{t_{k}}, Y_{t_{k}}, Z_{t_{k}}\right)\right) \frac{B_{s}-B_{t_{k}}}{s-t_{k}}\right]\right\| \\
& \leq\left\|f\left(s, B_{s}, Y_{s}, Z_{s}\right)-f\left(s, B_{t_{k}}, Y_{t_{k}}, Z_{t_{k}}\right)\right\| \frac{1}{\sqrt{s-t_{k}}} \\
& \leq C\left(T, L_{f}, C_{5.3}^{y}, C_{5.3}^{z}, p_{0}\right)(T-s)^{\frac{\alpha-2}{2}} \frac{h^{\frac{1}{2}}}{\sqrt{s-t_{k}}},
\end{aligned}
$$

where the last inequality follows in the same way as in (13). Consequently, we have

$$
\begin{aligned}
\left\|Z_{t_{k}}-Z_{t_{k}}^{n}\right\| \leq & \frac{C\left(C_{g}, T, p_{0}\right)}{\left(T-t_{k}\right)^{\frac{1}{2}}} h^{\frac{\alpha}{4}} \\
& +C\left(T, L_{f}, K_{f}, C_{5.3}^{y}, C_{5.3}^{z}, c_{5.4}^{1,2}, p_{0}\right) \int_{t_{k}}^{T} \frac{d s}{(T-s)^{1-\frac{\alpha}{2}}\left(s-t_{k}\right)^{\frac{3}{4}}} h^{\frac{1}{4}} \\
& +C\left(T, p_{0}, L_{f}, K_{f}, c_{5.4}\right) h \sum_{m=k+1}^{n-1} \frac{1}{\left(T-t_{m}\right)^{1-\frac{\alpha}{2}}} \frac{h^{\frac{1}{4}}}{\left(t_{m}-t_{k}\right)^{\frac{3}{4}}} \\
& +L_{f} h \sum_{m=k+1}^{n-1}\left(\left\|B_{t_{m}}-B_{t_{m}}^{n}\right\|+\left\|Y_{t_{m}}-Y_{t_{m}}^{n}\right\|+\left\|Z_{t_{m}}-Z_{t_{m}}^{n}\right\|\right) \frac{1}{\sqrt{t_{m-k}}} .
\end{aligned}
$$


Lemma A.2 enables to bound the second and third term of the r.h.s. by $C \frac{h^{\frac{1}{4}}}{\left(T-t_{k}\right)^{\frac{3}{4}-\frac{\alpha}{2}}} B\left(\frac{\alpha}{2}, \frac{1}{4}\right)$, which is bounded by $C \frac{h^{\frac{\alpha}{4}}}{\left(T-t_{k}\right)^{\frac{1}{2}-\frac{\alpha}{4}}}$. Thus, we get

$$
\left\|Z_{t_{k}}-Z_{t_{k}}^{n}\right\| \leq \frac{C_{0} h^{\frac{\alpha}{4}}}{\left(T-t_{k}\right)^{\frac{1}{2}}}+L_{f} h \sum_{m=k+1}^{n-1}\left(\left\|Y_{t_{m}}-Y_{t_{m}}^{n}\right\|+\left\|Z_{t_{m}}-Z_{t_{m}}^{n}\right\|\right) \frac{1}{\sqrt{t_{m-k}}}
$$

Then we use (14) and the above estimate to get

$$
\left\|Y_{t_{k}}-Y_{t_{k}}^{n}\right\|+\left\|Z_{t_{k}}-Z_{t_{k}}^{n}\right\| \leq \frac{C_{0} h^{\frac{\alpha}{4}}}{\left(T-t_{k}\right)^{\frac{1}{2}}}+C\left(L_{f}\right) h \sum_{m=k+1}^{n-1}\left(\left\|Y_{t_{m}}-Y_{t_{m}}^{n}\right\|+\left\|Z_{t_{m}}-Z_{t_{m}}^{n}\right\|\right) \frac{1}{\sqrt{t_{m-k}}}
$$

If this inequality is iterated, one gets a shape where the Gronwall lemma applies. Indeed, setting $a_{m}:=\left(\left\|Y_{t_{m}}-Y_{t_{m}}^{n}\right\|+\left\|Z_{t_{m}}-Z_{t_{m}}^{n}\right\|\right)$ one has to consider the double sum

$$
\sum_{m=k+1}^{n-1}\left(\sum_{l=m+1}^{n-1} a_{l} \frac{h}{\sqrt{t_{l-m}}}\right) \frac{h}{\sqrt{t_{m-k}}}=h \sum_{l=k+1}^{n-1}\left(\sum_{m=k+1}^{l-1} \frac{h}{\sqrt{t_{m-k}} \sqrt{t_{l-m}}}\right) a_{l} \leq C h \sum_{l=k+1}^{n-1} a_{l} .
$$

Consequently,

$$
\left\|Y_{t_{k}}-Y_{t_{k}}^{n}\right\|+\left\|Z_{t_{k}}-Z_{t_{k}}^{n}\right\| \leq \frac{C_{0} h^{\frac{\alpha}{4}}}{\left(T-t_{k}\right)^{\frac{1}{2}}}
$$

which gives the bound on the error on $Z$. Moreover, (14) yields

$$
\left\|Y_{t_{k}}-Y_{t_{k}}^{n}\right\| \leq C_{0} h^{\frac{\alpha}{4}}
$$

If $v \in\left[t_{k}, t_{k+1}\right)$, we have by Theorem 5.3 that

$$
\begin{aligned}
\left\|Y_{v}-Y_{v}^{n}\right\| & \leq\left\|Y_{v}-Y_{t_{k}}\right\|+\left\|Y_{t_{k}}-Y_{t_{k}}^{n}\right\| \leq C\left(C_{5.3}^{y}, T, p_{0}\right)\left(\int_{t_{k}}^{v}(T-r)^{\alpha-1} d r\right)^{\frac{1}{2}}+\left\|Y_{t_{k}}-Y_{t_{k}}^{n}\right\|, \\
\left\|Z_{v}-Z_{v}^{n}\right\| & \leq\left\|Z_{v}-Z_{t_{k}}\right\|+\left\|Z_{t_{k}}-Z_{t_{k}}^{n}\right\| \\
& \leq C\left(C_{5.3}^{z}, T, p_{0}\right)\left(\int_{t_{k}}^{v}(T-r)^{\alpha-2} d r\right)^{\frac{1}{2}}+\left\|Z_{t_{k}}-Z_{t_{k}}^{n}\right\|,
\end{aligned}
$$

where

$$
\int_{t_{k}}^{v}(T-r)^{\alpha-1} d r \leq \frac{1}{\alpha}\left(v-t_{k}\right)^{\alpha} \leq \frac{1}{\alpha} h^{\alpha}
$$


and

$$
\begin{aligned}
\int_{t_{k}}^{v}(T-r)^{\alpha-2} d r & \leq \frac{1}{(T-v)^{1-\frac{\alpha}{2}}} \int_{t_{k}}^{v}(T-r)^{\frac{\alpha}{2}-1} d r \leq \frac{1}{(T-v)^{1-\frac{\alpha}{2}}} \frac{2}{\alpha}\left(v-t_{k}\right)^{\frac{\alpha}{2}} \\
& \leq \frac{2}{\alpha} \frac{h^{\frac{\alpha}{2}}}{(T-v)^{1-\frac{\alpha}{2}}} .
\end{aligned}
$$

\section{Numerical simulations}

This section deals with the algorithm used to compute $\left(Y_{t_{k}}^{n}, Z_{t_{k}}^{n}\right)_{k=0, \ldots, n}$ and numerical experiments for different terminal conditions and drivers. In the first three cases the exact solution is available and we are able to compute the error $\left(Y^{n}-Y, Z^{n}-Z\right)$ in $L_{2}$-norm. In the last two cases the exact solution is unknown, therefore we plot the evolution of $\left(Y^{n}, Z^{n}\right)$ w.r.t. $n$.

\subsection{Simulation of $\left(\tau_{1}, \ldots, \tau_{n}\right)$ and $B^{n}$}

In order to simulate $\left(\tau_{1}, \ldots, \tau_{n}\right)$, we use the fact that

$$
\tau_{0}=0 \quad \text { and } \quad \forall k \geq 1, \quad \tau_{k}=\tau_{k-1}+\sigma_{k},
$$

where $\left(\sigma_{k}\right)_{1 \leq n}$ is an i.i.d. sequence whose common law $\sigma$ represents the first exit time of the Brownian motion $B$ of the interval $[-\sqrt{h}, \sqrt{h}]$,

$$
\sigma:=\inf \left\{t>0:\left|B_{t}\right|=\sqrt{h}\right\} .
$$

From the book of Borodin and Salminen [5], we have that the Laplace transform of $\sigma$ is given by $\mathbb{E}\left(e^{-\lambda \sigma}\right)=\frac{1}{\cosh (\sqrt{2 \lambda h})}$.

Let $F$ denote the cumulative distribution function of $\sigma$. It holds $\mathbb{E}\left(e^{-\lambda \sigma}\right)=\lambda \hat{F}(\lambda)$, where $\hat{F}$ is the Laplace transform of $F$. Then, to obtain $F$, it remains to inverse numerically its Laplace transform. Once we have $F$, we simulate the sequence $\left(\sigma_{k}\right)_{1 \leq k \leq n}$ by following the steps of Algorithm 1 .

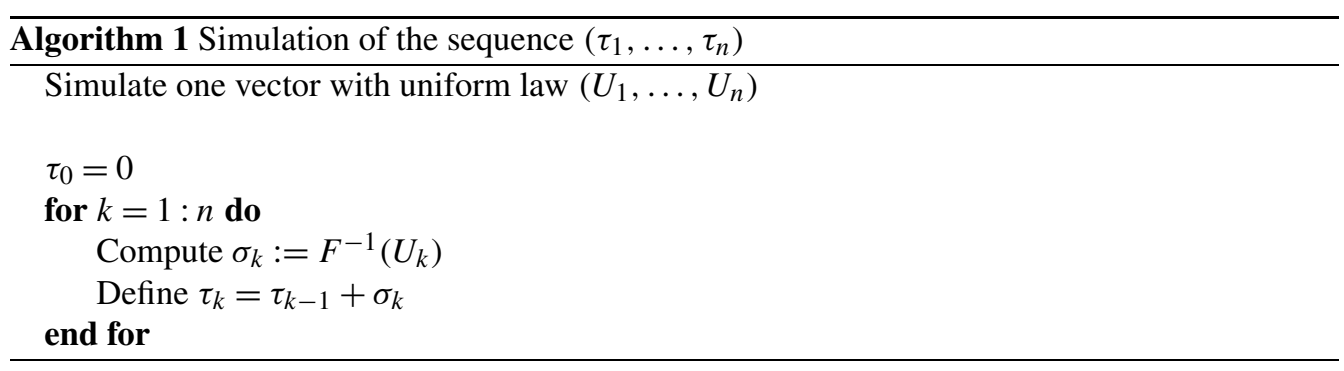




\subsection{Simulation of $B^{n}$}

In order to get the trajectory $B_{t_{1}}^{n}, \ldots, B_{t_{n}}^{n}\left(B_{t_{0}}^{n}=0\right)$, we simulate an i.i.d. Bernoulli sequence $\left(\xi_{k}\right)_{1 \leq k \leq n}$ that is, $\mathbb{P}\left(\xi_{k}= \pm 1\right)=\frac{1}{2}$. Then

$$
B_{t_{k+1}}^{n}= \begin{cases}B_{t_{k}}^{n}+\sqrt{h} & \text { if } \xi_{k}=1 \\ B_{t_{k}}^{n}-\sqrt{h} & \text { otherwise }\end{cases}
$$

\subsection{Simulation of $\left(Y^{n}, Z^{n}\right)$}

Since $B^{n}$ is built using the random walk (15), it can be represented by a recombining binomial tree. Both $\left(Y_{t_{k}}^{n}\right)_{0 \leq k \leq n}$ and $\left(Z_{t_{k}}^{n}\right)_{0 \leq k \leq n-1}$ can then also be represented as a recombining binomial tree. Since $Y_{t_{n}}^{n}=g\left(B_{t_{n}}^{n}\right)$, we solve backward in time the BSDE by following these equalities, ensuing from (2) ( $Y_{t_{k}}^{n}$ has been replaced by $Y_{t_{k+1}}^{n}$ in the generator term, but the error induced by this modification is smaller than the ones we consider)

$$
\left\{\begin{array}{l}
Z_{t_{k}}^{n}=\frac{1}{\sqrt{h}} \mathbb{E}_{\tau_{k}}\left(Y_{t_{k+1}}^{n} \varepsilon_{k+1}\right), \\
Y_{t_{k}}^{n}=\mathbb{E}_{\tau_{k}}\left(Y_{t_{k+1}}^{n}+h f\left(t_{k+1}, B_{t_{k}}^{n}, Y_{t_{k+1}}^{n}, Z_{t_{k}}^{n}\right)\right) .
\end{array}\right.
$$

Practically, we compute $\left(Y^{n}, Z^{n}\right)$ backward in time in the following way. From (15) it is easy to see that $B_{t_{k}}^{n}$ has $k+1$ different values. We store all values of $B^{n}$ in the upper triangular $(n+1) \times(n+1)$ matrix B defining $\mathrm{B}[i, k]=\sqrt{h}(k-2 i)(i$ and $k$ vary from 0 to $n)$. Similarly we store the values of $Y^{n}$ and $Z^{n}$ in the upper triangular $(n+1) \times(n+1)$ matrices $\mathrm{Y}$ and $\mathrm{Z}$ setting $\mathrm{Y}[i, k]=Y_{t_{k}}^{n}\left(B_{t_{k}}^{n}=\mathrm{B}[i, k]\right)$ and $\mathrm{z}[i, k]=Z_{t_{k}}^{n}\left(B_{t_{k}}^{n}=\mathrm{B}[i, k]\right)(i$ and $k$ vary from 0 to $n)$. Algorithm 2 describes the computation of the conditional expectations appearing in (16). Note that the computation of the conditional expectation is very simple: to get for instance the values of $\mathbb{E}_{\tau_{k}}\left(Y_{t_{k+1}}^{n}\right)$, we compute $\frac{1}{2}(\mathrm{Y}[i, k+1]+\mathrm{Y}[i+1, k+1])$ for $i=0, \ldots, k+1$.

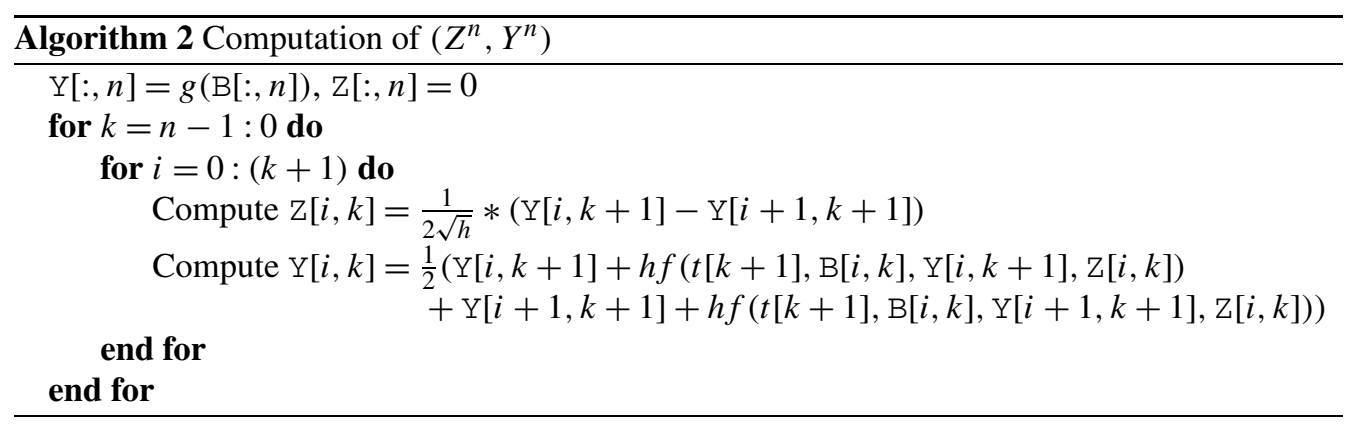




\subsection{Study of the error $\mathbb{E}\left|Y_{t_{k}}^{n}-Y_{t_{k}}\right|^{2}$ and $\mathbb{E}\left|Z_{t_{k}}^{n}-Z_{t_{k}}\right|^{2}$}

In this subsection, we assume that we are able to compute the exact solution $(Y, Z)$. We want to study numerically the convergence in $n$ of $\mathbb{E}\left|Y_{t_{k}}^{n}-Y_{t_{k}}\right|^{2}$ and $\mathbb{E}\left|Z_{t_{k}}^{n}-Z_{t_{k}}\right|^{2}$, where $(Y, Z)$ solves (1) and $\left(Y^{n}, Z^{n}\right)$ solves (6). To do so, we approximate the error $\mathbb{E}\left|A_{t_{k}}^{n}-A_{t_{k}}\right|^{2}(A=Y$ or $A=Z)$ by Monte Carlo:

$$
\mathbb{E}\left|A_{t_{k}}^{n}-A_{t_{k}}\right|^{2} \sim \frac{1}{M} \sum_{m=1}^{M}\left|A_{t_{k}}^{n, m}-A_{t_{k}}^{m}\right|^{2}:=E_{A}
$$

1. For each Monte Carlo simulation, we pick at random one sequence $\left(\xi_{1}, \ldots, \xi_{n}\right)$ (which gives the value of $\left.\left(B_{t_{1}}^{n}, \ldots, B_{t_{n}}^{n}\right)\right)$ and one sequence $\left(\tau_{1}, \ldots, \tau_{n}\right)$.

2. From the sequence $\left(\xi_{1}, \ldots, \xi_{n}\right)$ we get the trajectory of $Y^{n}$, including $Y_{t_{k}}^{n}$.

3. From the sequence $\left(B_{\tau_{1}}, \ldots, B_{\tau_{n}}\right)$ (which is equal to $\left(B_{t_{1}}^{n}, \ldots, B_{t_{n}}^{n}\right)$ ), we compute $B_{t_{k}}$ by using the Brownian bridge method. We deduce $\left(Y_{t_{k}}, Z_{t_{k}}\right)$ as functions of $B_{t_{k}}$.

In the following experiments, we plot the logarithm of the errors $E_{Y}$ and $E_{Z}$ (defined in (17)) w.r.t. $\log (n)$. From Theorem 3.1, we get that $\log \left(E_{Y}\right)$ and $\log \left(E_{Z}\right)$ decrease as $-\frac{\alpha}{2} \log (n)$. By using a linear regression, we compute the slope of the line solving the least square problem and compare it to $-\frac{\alpha}{2}$.

\subsection{Numerical experiment}

4.5.1. Case $g(x)=e^{T+x}$ and $f(y, z)=y+z$

We consider the BSDE with terminal condition $g(x)=e^{T+x}$ and driver $f(y, z)=y+z$. In this case, we know that $Y_{t}=e^{T+B_{t}+\frac{5}{2}(T-t)}$. We run $M=20,000$ Monte Carlo simulations. We fix $T=1$.

Figure 1 represents $\log ($ error on $Y$ ) and $\log ($ error on $Z$ ) at time $t=0.5$ (the error is defined by (17)) with respect to $\log (n)$, when $n$ varies between 10 to 70 with step 10 . For the $Y$ case, the slope ensuing from the linear regression is -0.53 . Even though $g(x)=e^{T+x}$ does not satisfy (3), $g$ is locally Lipschitz continuous, and the outcome seems to be consistent with Theorem 3.1 for $\alpha=1$. For the $Z$ case, we get the slope -0.61 .

\subsubsection{Case $g(x)=x^{2}$ and $f(y, z)=y+z$}

In that case, we know that $Y_{t}=e^{T-t}\left(\left(B_{t}-(T-t)\right)^{2}+T-t\right)$ and $Z_{t}=2 e^{T-t}\left(B_{t}-(T-t)\right)$. We run $M=20,000$ Monte Carlo simulations. We fix $T=1$.

Figure 2 represents $\log$ (error on $Y$ ) and $\log$ (error on $Z$ ) with respect to $\log (n)$ at time $t=0.5$ when $n$ varies between 10 to 100 with step 10 and from 200 to 500 with step 100 . The slope of the linear regression for $Y$ (resp. for $Z$ ) is -0.465 (resp. -0.48). The results are then consistent with Theorem 3.1. 

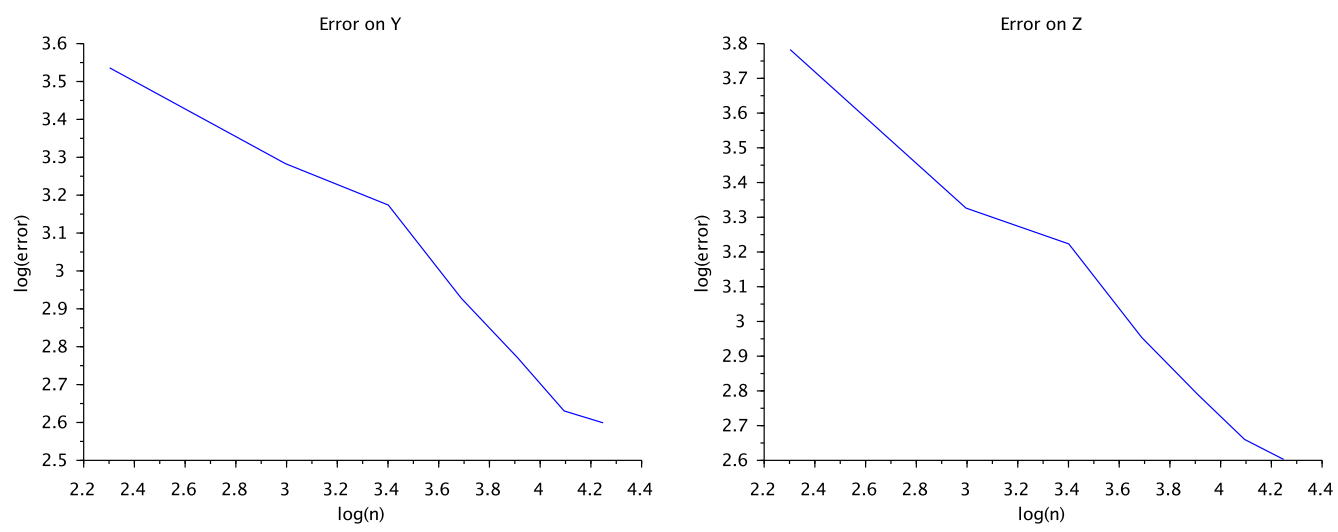

Figure 1. $\log ($ error on $Y)$ and $\log ($ error on $Z)$ at time $t=0.5$ w.r.t. $\log (n)-f(y, z)=y+z-g(x)=e^{1+x}$.
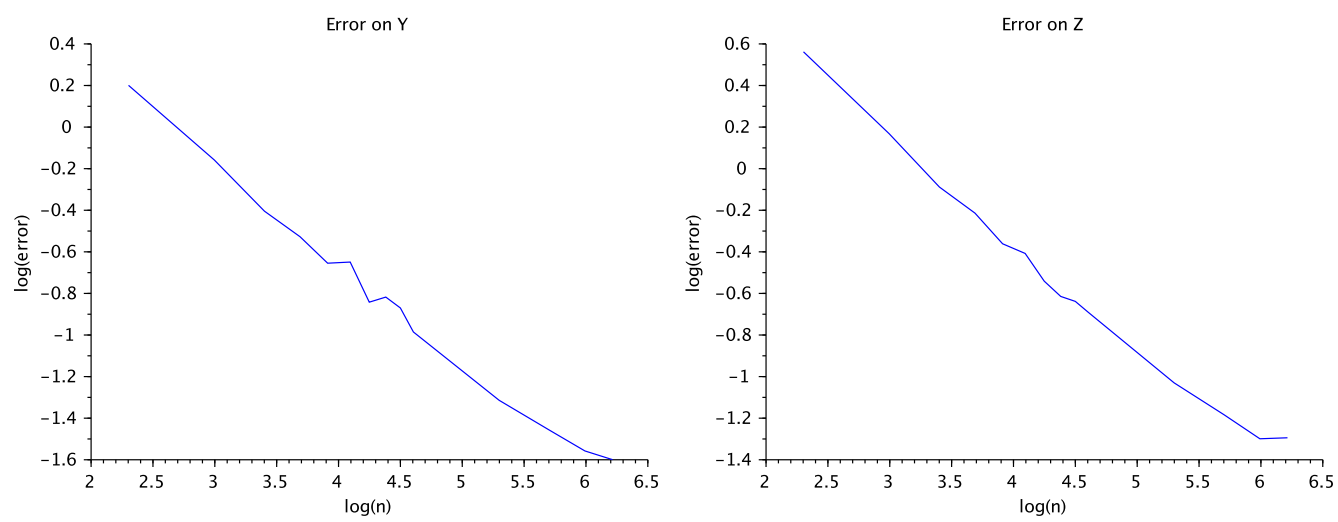

Figure 2. $\log$ (error on $Y$ ) (on the left) and $\log$ (error on $Z$ ) (on the right) at time $t=0.5$ as a function of $\log (n)-f(y, z)=y+z-g(x)=x^{2}$.

\subsubsection{Case $g(x)=\sqrt{|x|}$ and $f(y, z)=y+z$}

In that case, we know that $Y_{t}=e^{\frac{T-t}{2}} \tilde{\mathbb{E}}\left(\sqrt{\left|\tilde{B}_{T-t}+B_{t}\right|} e^{\tilde{B}_{T-t}}\right)$. We run $M=20,000$ Monte Carlo simulations. We fix $T=1$.

Figure 3 represents $\log ($ error on $Y$ ) at time $t=0.5$ and at time $t=0$ with respect to $\log (n)$ when $n$ varies between 10 to 100 with step 10 and from 200 to 500 by step 100 . The slope of the linear regression is -0.51 (resp. -1.7 ) when $t=0.5$ (resp. when $t=0$ ). Here we notice that the modulus of the slope we get is larger than $\frac{1}{4}$, the upper bound obtained in that case in Theorem 3.1. 

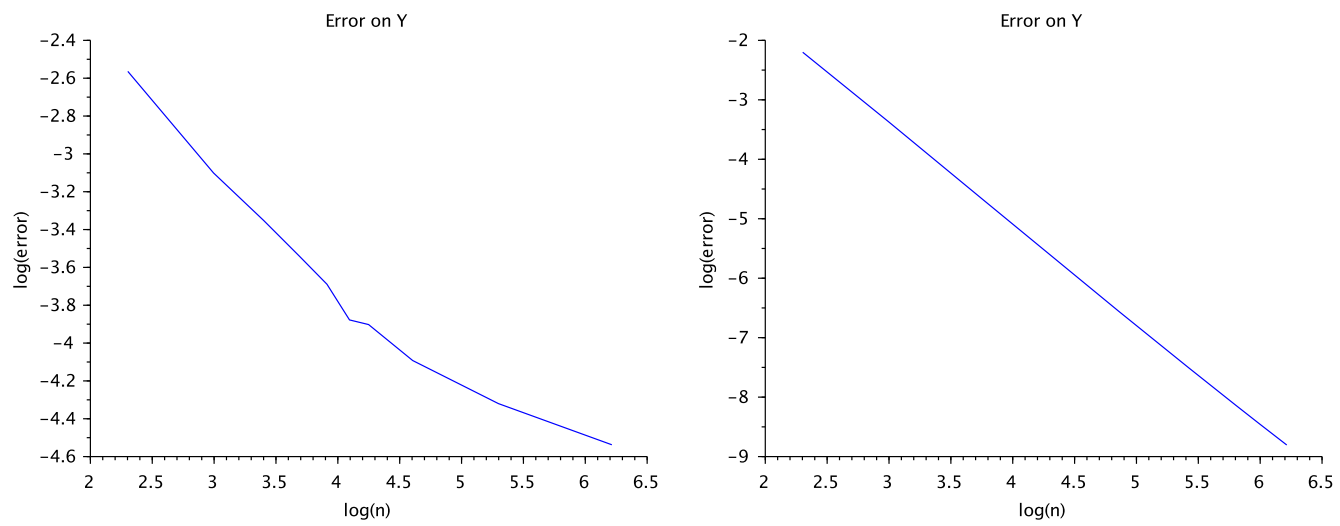

Figure 3. $\log$ (error on $Y$ ) at time $t=0.5$ (on the left) and at time $t=0$ (on the right) as a function of $\log (n)-f(y, z)=y+z-g(x)=\sqrt{|x|}$.

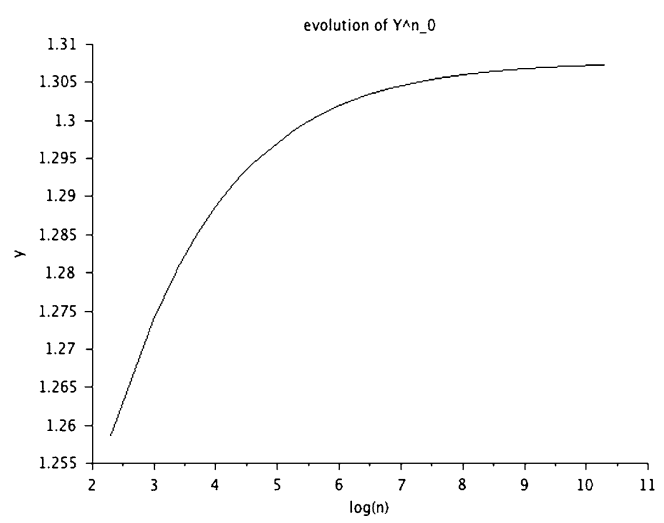

Figure 4. Evolution of $Y_{0}^{n}$ as a function of $\log (n)-f(y, z)=\cos (y)-g(x)=|x|^{1 / 4}$.

4.5.4. Case $g(x)=|x|^{1 / 4}$ and $f(y, z)=\cos (y)$

There is no explicit solution. We fix $T=1$ and plot the evolution of $Y_{0}^{n}$ for different values of $n$ where $n$ varies from 10 to 100 by step 10 , then from 100 to 1000 by step 100, from 2000 to 10,000 by step 1000 and the last three values are $15,000,20,000$ and 30,000. We notice a slow convergence (in $n$ ) of $Y_{0}^{n}$ which can be expected to happen in view of Theorem 3.1.

4.5.5. Case $g(x)=x^{2}$ and $f(y, z)=\cos (y)+\sin (z)$

There is no explicit solution. We fix $T=1$ and plot the evolution of $Y_{0}^{n}$ and $Z_{0}^{n}$ for different values of $n$ (see Figure 5), where $n$ varies from 10 to 100 by step 10 . The convergence of $Y_{0}^{n}$ and $Z_{0}^{n}$ in $n$ is quite fast. 

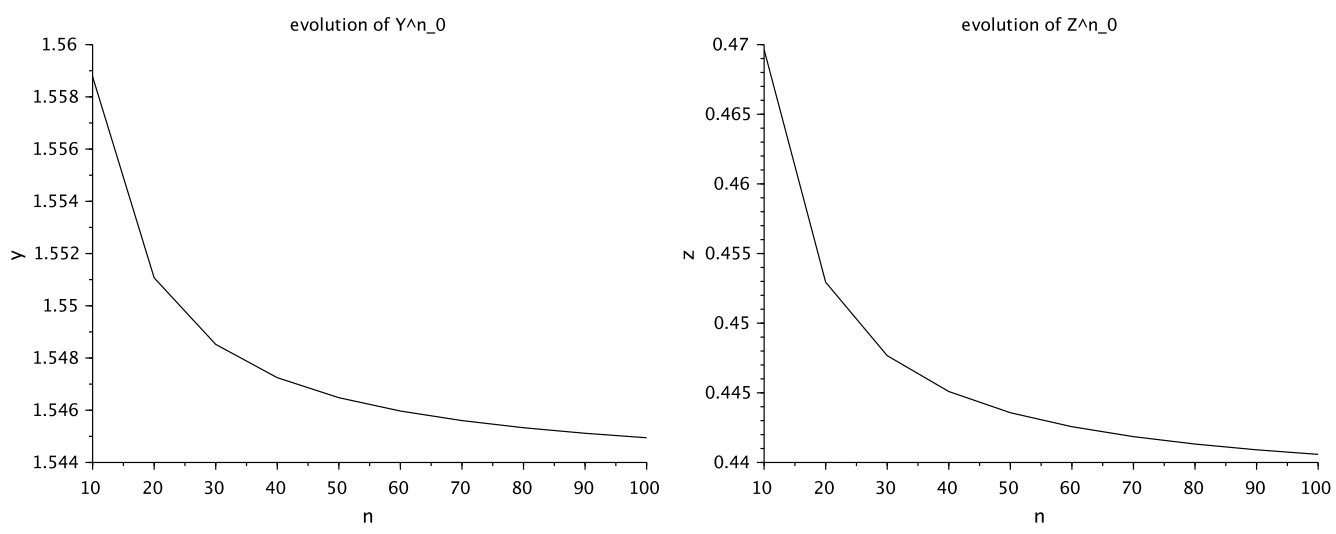

Figure 5. Evolution of $Y_{0}^{n}$ (on the left) and $Z_{0}^{n}$ (on the right) as a function of $n-f(y, z)=\cos (y)+\sin (z)-$ $g(x)=x^{2}$.

\section{Some properties of solutions to PDEs and BSDEs}

In the following, we recall and prove results for FBSDEs with a general forward process, even though we apply them in the present paper only for the case where the forward process is just the Brownian motion. Restricting ourselves to the case of Brownian motion would not shorten the proofs considerably. Let us consider the following SDE started in $(t, x)$,

$$
X_{s}^{t, x}=x+\int_{t}^{s} b\left(r, X_{r}^{t, x}\right) d r+\int_{t}^{s} \sigma\left(r, X_{r}^{t, x}\right) d B_{r}, \quad 0 \leq t \leq s \leq T,
$$

where $b$ and $\sigma$ satisfy the following.

\section{Assumption 5.1.}

1. $b, \sigma \in C_{b}^{0,2}([0, T] \times \mathbb{R})$, in the sense that the derivatives of order $k=0,1,2$ w.r.t. the space variable are continuous and bounded on $[0, T] \times \mathbb{R}$,

2. the first and second derivatives of $b$ and $\sigma$ w.r.t. the space variable are assumed to be $\gamma$-Hölder continuous (for some $\gamma \in(0,1]$, w.r.t. the parabolic metric $d\left((x, t),\left(x^{\prime}, t^{\prime}\right)\right)=$ $\left(\left|x-x^{\prime}\right|^{2}+\left|t-t^{\prime}\right|\right)^{\frac{1}{2}}$ on all compact subsets of $[0, T] \times \mathbb{R}$,

3. $b, \sigma$ are $\frac{1}{2}$-Hölder continuous in time, uniformly in space,

4. $\sigma(t, x) \geq \delta>0$ for all $(t, x)$.

\subsection{Malliavin weights}

In this section, we recall the Malliavin weights and their properties from [22], Section 1.1 and Remark 3. 
Lemma 5.2. Let $H: \mathbb{R} \rightarrow \mathbb{R}$ be a polynomially bounded Borel function. If Assumption 5.1 holds and $X^{t, x}$ is given by (18), then setting

$$
G(t, x):=\mathbb{E} H\left(X_{R}^{t, x}\right)
$$

implies that $G \in C^{1,2}([0, R) \times \mathbb{R})$. Especially it holds for $0 \leq t \leq r<R \leq T$ that

$$
\partial_{x} G\left(r, X_{r}^{t, x}\right)=\mathbb{E}\left[H\left(X_{R}^{t, x}\right) N_{R}^{r, 1,(t, x)} \mid \mathcal{F}_{r}^{t}\right], \quad \text { and } \quad \partial_{x}^{2} G\left(r, X_{r}^{t, x}\right)=\mathbb{E}\left[H\left(X_{R}^{t, x}\right) N_{R}^{r, 2,(t, x)} \mid \mathcal{F}_{r}^{t}\right],
$$

where $\left(\mathcal{F}_{r}^{t}\right)_{r \in[t, T]}$ is the augmented natural filtration of $\left(B_{r}^{t, 0}\right)_{r \in[t, T]}$,

$$
\begin{aligned}
N_{R}^{r, 1,(t, x)} & =\frac{1}{R-r} \int_{r}^{R} \frac{\nabla X_{s}^{t, x}}{\sigma\left(s, X_{s}^{t, x}\right) \nabla X_{r}^{t, x}} d B_{S} \quad \text { and } \\
N_{R}^{r, 2,(t, x)} & =\frac{N_{R}^{\rho, 1,(t, x)} \nabla X_{R}^{t, x} N_{\rho}^{r, 1,(t, x)}+\nabla N_{R}^{\rho, 1,(t, x)}}{\nabla X_{r}^{t, x}},
\end{aligned}
$$

with $\rho:=\frac{r+R}{2}$. Moreover, for $q \in(0, \infty)$ it holds a.s.

$$
\left(\mathbb{E}\left[\left|N_{R}^{r, i,(t, x)}\right|^{q} \mid \mathcal{F}_{r}^{t}\right]\right)^{\frac{1}{q}} \leq \frac{\kappa_{q}}{(R-r)^{\frac{i}{2}}},
$$

and $\mathbb{E}\left[N_{R}^{r, i,(t, x)} \mid \mathcal{F}_{r}^{t}\right]=0$ a.s. for $i=1$, 2. Finally, we have

$$
\left\|\partial_{x} G\left(r, X_{r}^{t, x}\right)\right\|_{L_{p}(\mathbb{P})} \leq \kappa_{q} \frac{\left\|H\left(X_{R}^{t, x}\right)-\mathbb{E}\left[H\left(X_{R}^{t, x}\right) \mid \mathcal{F}_{r}^{t}\right]\right\|_{L_{p}(\mathbb{P})}}{\sqrt{R-r}}
$$

and

$$
\left\|\partial_{x}^{2} G\left(r, X_{r}^{t, x}\right)\right\|_{L_{p}(\mathbb{P})} \leq \kappa_{q} \frac{\left\|H\left(X_{R}^{t, x}\right)-\mathbb{E}\left[H\left(X_{R}^{t, x}\right) \mid \mathcal{F}_{r}^{t}\right]\right\|_{L_{p}(\mathbb{P})}}{R-r}
$$

for $1<q, p<\infty$ with $\frac{1}{p}+\frac{1}{q}=1$.

\subsection{Regularity of solutions to BSDEs}

Let us now consider the FBSDE

$$
Y_{s}^{t, x}=g\left(X_{T}^{t, x}\right)+\int_{s}^{T} f\left(r, X_{r}^{t, x}, Y_{r}^{t, x}, Z_{r}^{t, x}\right) d r-\int_{s}^{T} Z_{r}^{t, x} d B_{r}, \quad 0 \leq t \leq s \leq T,
$$

where $X^{t, x}$ is the process satisfying (18). The following result is taken from [22], Theorem 1 . We reformulate it here for the simple situation where we need it. On the other hand, we will use $\mathbb{P}_{t, x}$ and are interested in an estimate for all $(t, x) \in[0, T) \times \mathbb{R}$. 
Theorem 5.3. Let Assumption 2.1 and 5.1 hold. Then for any $p \in[2, \infty)$ the following assertions are true.

(i) There exists a constant $C_{5.3}^{y}>0$ such that for $0 \leq t<s<T$ and $x \in \mathbb{R}$,

$$
\left\|Y_{S}-Y_{t}\right\|_{L_{p}\left(\mathbb{P}_{t, x}\right)} \leq C_{5.3}^{y} \Psi(x)\left(\int_{t}^{s}(T-r)^{\alpha-1} d r\right)^{\frac{1}{2}} .
$$

(ii) There exists a constant $C_{5.3}^{z}>0$ such that for $0 \leq t<s<T$ and $x \in \mathbb{R}$,

$$
\left\|Z_{s}-Z_{t}\right\|_{L_{p}\left(\mathbb{P}_{t, x}\right)} \leq C_{5.3}^{z} \Psi(x)\left(\int_{t}^{s}(T-r)^{\alpha-2} d r\right)^{\frac{1}{2}} .
$$

The constants $C_{5.3}^{y}$ and $C_{5.3}^{z}$ depend on $K_{f}, L_{f}, C_{g}, c_{5.4}^{1,2}, T, p_{0}, b, \sigma, \kappa_{q}$ and $p$.

Proof of Theorem 5.3. (i) First, we follow the step [22], Theorem 1, proof of $\left(C 2_{l}\right) \Longrightarrow\left(C 3_{l}\right)$. We conclude from the linear growth $|f(r, x, y, z)| \leq L_{f}(|x|+|y|+|z|)+K_{f}$ and from the Burkholder-Davis-Gundy inequality with constant $a_{p}>0$ that

$$
\begin{aligned}
\left\|Y_{s}-Y_{t}\right\|_{L_{p}\left(\mathbb{P}_{t, x}\right)} & \|\| \int_{t}^{s} f\left(r, X_{r}, Y_{r}, Z_{r}\right) d r-\int_{t}^{s} Z_{r} d B_{r} \|_{L_{p}\left(\mathbb{P}_{t, x}\right)} \\
\leq & K_{f}(s-t)+L_{f} \int_{t}^{s}\left\|X_{r}\right\|_{L_{p}\left(\mathbb{P}_{t, x}\right)}+\left\|Y_{r}\right\|_{L_{p}\left(\mathbb{P}_{t, x}\right)} \\
& +\left\|Z_{r}\right\|_{L_{p}\left(\mathbb{P}_{t, x}\right)} d r+a_{p}\left(\int_{t}^{s}\left\|Z_{r}\right\|_{L_{p}\left(\mathbb{P}_{t, x}\right)}^{2} d r\right)^{\frac{1}{2}} .
\end{aligned}
$$

We then use (i) and (ii) of Theorem 5.4 below to get

$$
\begin{aligned}
\| Y_{s}- & Y_{t} \|_{L_{p}\left(\mathbb{P}_{t, x}\right)} \\
\leq & K_{f}(s-t) \\
& \quad+C\left(T, L_{f}, c_{5.4}^{1,2}, p, b, \sigma, p_{0}\right) \Psi(x)\left[\int_{t}^{s}\left(1+(T-r)^{\frac{\alpha-1}{2}}\right) d r+\left(\int_{t}^{s}(T-r)^{\alpha-1} d r\right)^{\frac{1}{2}}\right] .
\end{aligned}
$$

(ii) Here one can follow [22], Theorem 1 , proof of $\left(C 4_{l}\right) \Longrightarrow\left(C 1_{l}\right)$.

Step 1: We first assume additionally that $f:[0, T] \times \mathbb{R}^{3} \rightarrow \mathbb{R}$ is continuously differentiable in $x, y$, and $z$ with uniformly bounded derivatives as it was assumed for [22], Theorem 1 . To take the dependency on $x$ into consideration which arises since we use $\mathbb{P}_{t, x}$, it suffices to replace everywhere in the proof in [22] the constant $c_{B_{p, \infty}^{\Theta}}$ by $C\left(T, C_{g}, \sigma, b, p, p_{0}\right) \Psi(x)$. The constant $C_{5.3}^{z}$ depends moreover on $L_{f}$ and $\kappa_{q}$. 
Step 2: Now let $f$ be as in Assumption 5.1. In [22], Theorem 1, proof of $\left(C 4_{l}\right) \Longrightarrow\left(C 1_{l}\right)$, a linear BSDE is used which describes the behaviour of the process $\mathrm{Z}$ minus its counterpart where the generator is identically 0 . Here the partial derivatives of $f_{x}, f_{y}, f_{z}$ appear but only their uniform bound is needed in the estimates. Hence, if $f$ satisfies (4), we can use mollifying as explained in (26) below (one may choose $N=\infty$ ). Since $\left|\partial_{x} f^{\varepsilon}(t, x, y, z)\right|,\left|\partial_{y} f^{\varepsilon}(t, x, y, z)\right|$ and $\left|\partial_{z} f^{\varepsilon}(t, x, y, z)\right|$ are bounded by $L_{f}$ we conclude from Step 1 that for all $\varepsilon>0$ the process $Z^{\varepsilon}$ corresponding to $f^{\varepsilon}$ satisfies

$$
\left\|Z_{s}^{\varepsilon}-Z_{t}^{\varepsilon}\right\|_{L_{p}\left(\mathbb{P}_{t, x}\right)} \leq C_{5.3}^{z} \Psi(x)\left(\int_{t}^{s}(T-r)^{\alpha-2} d r\right)^{\frac{1}{2}}
$$

for $p \geq 2$. Especially, the family $\left\{\left|Z_{s}^{\varepsilon}-Z_{t}^{\varepsilon}\right|^{q}: \varepsilon>0\right\}$ is then uniformly integrable provided that $q<p$. By an a priori estimate (cf. [8], Lemma 3.1) we have that

$$
\mathbb{E} \int_{0}^{T}\left|Z_{r}-Z_{r}^{\varepsilon}\right|^{2} d r \leq C \int_{0}^{T} \sup _{x, y, z}\left|f(r, x, y, z)-f^{\varepsilon}(r, x, y, z)\right|^{2} d r \leq C \varepsilon^{2} T L_{f}^{2} .
$$

Fubini's theorem implies that there exists a sequence $\varepsilon_{m} \rightarrow 0$ and a measurable set $N \subseteq[0, T]$ of Lebesgue measure zero, such that $\lim _{m \rightarrow \infty} \mathbb{E}\left|Z_{r}-Z_{r}^{\varepsilon_{m}}\right|^{2}=0$ for all $r \in[0, T] \backslash N$. Consequently, for any $q<p$ and all $t, s \in[0, T] \backslash N$ with $t<s$,

$$
\left\|Z_{s}-Z_{t}\right\|_{L_{q}\left(\mathbb{P}_{t, x}\right)} \leq C_{5.3}^{z} \Psi(x)\left(\int_{t}^{s}(T-r)^{\alpha-2} d r\right)^{\frac{1}{2}} .
$$

The assertion follows for all $q \geq 2$ since (21) holds for all $p \in[2, \infty)$. Since by Theorem 5.4(ii) the process $Z$ does have a continuous version, we finally get the assertion for all $t<s$.

\subsection{Properties of the associated PDE}

We collect in the theorem below properties of the solution to the PDE which are mainly known. The new part concerns $\partial_{x}^{2} u$. For Lipschitz continuous $g$, the behaviour of $\partial_{x}^{2} u$ has been studied in [35]. General results related to this topic can be found in [19].

Theorem 5.4. Consider the FBSDE (20) and let Assumptions 2.1 and 5.1 hold. Then for the solution $u$ of the associated PDE

$$
\left\{\begin{array}{l}
u_{t}(t, x)+\frac{\sigma^{2}(t, x)}{2} u_{x x}(t, x)+b(t, x) u_{x}(t, x)+f\left(t, x, u(t, x), \sigma(t, x) u_{x}(t, x)\right)=0, \\
\quad t \in[0, T), x \in \mathbb{R}, \\
u(T, x)=g(x), \quad x \in \mathbb{R}
\end{array}\right.
$$


we have

(i) $Y_{t}=u\left(t, X_{t}\right)$ where $u(t, x)=\mathbb{E}_{t, x}\left(g\left(X_{T}\right)+\int_{t}^{T} f\left(r, X_{r}, Y_{r}, Z_{r}\right) d r\right)$ and $|u(t, x)| \leq$ $c_{5.4}^{1} \Psi(x)$ with $\Psi$ given in (5), where $c_{5.4}^{1}$ depends on $C_{g}, T, p_{0}, L_{f}, K_{f}$ and on the bounds and Lipschitz constants of $b$ and $\sigma$.

(ii) $u_{x}$ exists,

$$
u_{x}(t, x)=\mathbb{E}_{t, x}\left(g\left(X_{T}\right) N_{T}^{t, 1}+\int_{t}^{T} f\left(r, X_{r}, Y_{r}, Z_{r}\right) N_{r}^{t, 1} d r\right)
$$

and

(a) $u_{x}$ is continuous in $[0, T) \times \mathbb{R}$,

(b) $Z_{s}^{t, x}=u_{x}\left(s, X_{s}^{t, x}\right) \sigma\left(s, X_{s}^{t, x}\right)$,

(c) $\left|u_{x}(t, x)\right| \leq \frac{c_{5.4}^{2} \Psi(x)}{(T-t)^{\frac{1-\alpha}{2}}}$,

where $c_{5.4}^{2}$ depends on $C_{g}, T, p_{0}, \kappa_{2}, L_{f}, K_{f}$ and on the bounds and Lipschitz constants of $b$ and $\sigma$.

(iii) $u_{x x}$ exists,

$$
\begin{aligned}
u_{x x}(t, x)= & \mathbb{E}_{t, x}\left(g\left(X_{T}\right) N_{T}^{t, 2}\right. \\
& \left.+\int_{t}^{T}\left[f\left(r, X_{r}, Y_{r}, Z_{r}\right)-f\left(r, X_{t}, Y_{t}, Z_{t}\right)\right] N_{r}^{t, 2} d r\right),
\end{aligned}
$$

and

(a) $u_{x x}$ is continuous in $[0, T) \times \mathbb{R}$,

(b) $\left|u_{x x}(t, x)\right| \leq \frac{c_{5.4}^{3} \Psi(x)}{(T-t)^{1-\frac{\alpha}{2}}}$,

where $c_{5.4}^{3}$ depends on $C_{g}, T, p_{0}, \kappa_{2}, L_{f}, C_{5.3}^{y}, C_{5.3}^{z}$ and on the bounds and Lipschitz constants of $b$ and $\sigma$.

In the following $c_{5.4}$ represents $\left(c_{5.4}^{1}, c_{5.4}^{2}, c_{5.4}^{3}\right)$ and $c_{5.4}^{i, j}(i \neq j)$ represents $\left(c_{5.4}^{i}, c_{5.4}^{j}\right),(i, j) \in$ $\{1,2,3\}$.

Proof. (i): This follows from [37], Theorem 3.2.

(ii): From the proof of [37], Theorem 3.2, we get (22). The points (ii)(a) and (b) ensue from [37], Theorem 3.2(i). It remains to prove (c).

Proof of (ii)(c). We show the assertion for a generator not depending on $X$, since the terms arising from that dependency would be easy to treat. Since $\mathbb{E}_{t, x}\left(\mathbb{E}_{t, x}\left(g\left(X_{T}\right)\right) N_{T}^{t, 1}\right)=0$ we can subtract it from the right-hand side of (22) and get

$$
\partial_{x} u(t, x)=\mathbb{E}_{t, x}\left(\left[g\left(X_{T}\right)-\mathbb{E}_{t, x}\left(g\left(X_{T}\right)\right)\right] N_{T}^{t, 1}+\int_{t}^{T} f\left(r, Y_{r}, Z_{r}\right) N_{r}^{t, 1} d r\right) .
$$


It holds

$$
\mathbb{E}_{t, x}\left|g\left(X_{T}\right)-\mathbb{E}_{t, x} g\left(X_{T}\right)\right|^{2}=\mathbb{E}_{t, x}\left|g\left(X_{T}\right)-\tilde{\mathbb{E}} g\left(\tilde{X}_{T}^{t, X_{t}}\right)\right|^{2} \leq \mathbb{E}_{t, x} \tilde{\mathbb{E}}\left|g\left(X_{T}\right)-g\left(\tilde{X}_{T}^{t, X_{t}}\right)\right|^{2},
$$

and thanks to the Cauchy-Schwarz inequality with $\Psi_{1}=C_{g}\left(1+\left|X_{T}\right|^{p_{0}}+\left|\tilde{X}_{T}^{t, X_{t}}\right|^{p_{0}}\right)$ and equation (3),

$$
\begin{aligned}
\mathbb{E}_{t, x} \tilde{\mathbb{E}}\left|g\left(X_{T}\right)-g\left(\tilde{X}_{T}^{t, X_{t}}\right)\right|^{2} & \leq \mathbb{E}_{t, x} \tilde{\mathbb{E}}\left(\Psi_{1}^{2}\left|X_{T}-\tilde{X}_{T}^{t, X_{t}}\right|^{2 \alpha}\right) \\
& \leq\left[\mathbb{E}_{t, x} \tilde{\mathbb{E}} \Psi_{1}^{4}\right]^{\frac{1}{2}}\left[\mathbb{E}_{t, x} \tilde{\mathbb{E}}\left|X_{T}-\tilde{X}_{T}^{t, X_{t}}\right|^{4 \alpha}\right]^{\frac{1}{2}} \\
& \leq C\left(C_{g}, T, p_{0}, b, \sigma\right) \Psi^{2}(x)(T-t)^{\alpha} .
\end{aligned}
$$

Relation (19) and the Lipschitz continuity of $f$ imply

$$
\begin{aligned}
& \left|\partial_{x} u(t, x)\right| \\
& \leq \frac{C\left(C_{g}, T, p_{0}, \kappa_{2}, b, \sigma\right) \Psi(x)}{(T-t)^{\frac{1-\alpha}{2}}} \\
& \quad+C\left(L_{f}, K_{f}\right) \mathbb{E}_{t, x} \int_{t}^{T}\left(1+\left|u\left(r, X_{r}\right)\right|+\left|\partial_{x} u\left(r, X_{r}\right) \sigma\left(r, X_{r}\right)\right|\right)\left|N_{r}^{t, 1}\right| d r .
\end{aligned}
$$

Since we have $|g(x)| \leq \Psi(x)$, [37], Theorem 3.2(ii), gives $|u(t, x)| \leq c \Psi(x)$ and $\left|\partial_{x} u(t, x)\right| \leq$ $c \Psi(x)(T-t)^{-1 / 2}$, where c depends on $T, L_{f}, K_{f}, \kappa_{2}, b, \sigma$ and $p_{0}$. Hence, inequality (25) becomes

$$
\begin{aligned}
\left|\partial_{x} u(t, x)\right| \leq & \frac{C\left(C_{g}, T, p_{0}, \kappa_{2}, b, \sigma\right) \Psi(x)}{(T-t)^{\frac{1-\alpha}{2}}} \\
& +C\left(L_{f}, K_{f}, c, \sigma\right) \mathbb{E}_{t, x}\left(\int_{t}^{T}\left(1+\Psi\left(X_{r}\right)+\frac{\Psi\left(X_{r}\right)}{(T-r)^{\frac{1}{2}}}\right)\left|N_{r}^{t, 1}\right| d r\right) \\
\leq & \frac{C\left(C_{g}, T, p_{0}, \kappa_{2}, b, \sigma\right) \Psi(x)}{(T-t)^{\frac{1-\alpha}{2}}} \\
& +C\left(T, L_{f}, K_{f}, \kappa_{2}, b, \sigma, p_{0}\right) \int_{t}^{T} \frac{\Psi(x)}{(T-r)^{\frac{1}{2}}(r-t)^{\frac{1}{2}}} d r \\
\leq & \frac{C\left(C_{g}, T, p_{0}, \kappa_{2}, L_{f}, K_{f}, b, \sigma\right) \Psi(x)}{(T-t)^{\frac{1-\alpha}{2}}} .
\end{aligned}
$$

(iii): We start with an approximation of $g$ and $f$ by smooth and bounded functions. Let $\phi$ be a non-negative $C^{\infty}$ function with support $[-1,1]$, such that $\int_{\mathbb{R}} \phi(u) d u=1$, and $\varepsilon \in(0,1]$. For $N \in \mathbb{N}$ let $b_{N}: \mathbb{R} \rightarrow[-N-1, N+1]$ be a monotone $C^{\infty}$ function such that $0 \leq b_{N}^{\prime}(x) \leq 1$ 
and

$$
b_{N}(x):= \begin{cases}N+1, & x>N+2 \\ x, & |x| \leq N \\ -N-1, & x<-N-2\end{cases}
$$

Define

$$
g^{\varepsilon, N}(x)=\int_{-1}^{1} \phi(u) g\left(b_{N}(x)-\varepsilon u\right) d u
$$

and

$$
f^{\varepsilon, N}(r, y, z)=\int_{-1}^{1} \int_{-1}^{1} \phi(u) \phi(v) f\left(r, b_{N}(y)-\varepsilon u, b_{N}(z)-\varepsilon v\right) d u d v .
$$

Lemma 5.5. $g^{\varepsilon, N}$ and $f^{\varepsilon, N}$ satisfy

(a) $\left\|g^{\varepsilon, N}\right\|_{\infty}+\left\|f^{\varepsilon, N}\right\|_{\infty} \leq C=C(\varepsilon, N)$ for some $C(\varepsilon, N)>0$,

(b) $g^{\varepsilon, N}$ and $f^{\varepsilon, N}$ are $C^{\infty}$ functions, with bounded derivatives (the bounds depend on $\varepsilon$ and $N$ ). Moreover, $f^{\varepsilon, N}$ is a Lipschitz function in $y$ and $z$, with Lipschitz constant $L_{f}$,

(c) $g^{\varepsilon, N}$ satisfies (3), uniformly in $\varepsilon \in(0,1)$ and $N \geq 1$,

(d) for all $x \in \mathbb{R}$ and $\varepsilon \in[0,1]$, we have $\left|g^{\varepsilon, N}(x)-g(x)\right| \leq C\left(C_{g}\right) \Psi(x)\left(\varepsilon^{\alpha}+\frac{|x|^{\alpha+1}}{N}\right)$,

(e) for all $r \in[0, T]$ and for all $(y, z) \in \mathbb{R}^{2}$, we have

$$
\left|f^{\varepsilon, N}(r, y, z)-f(r, y, z)\right| \leq L_{f}\left(2 \varepsilon+\left|b_{N}(y)-y\right|+\left|b_{N}(z)-z\right|\right) .
$$

\section{Proof.}

(a) Since $g$ is locally Hölder continuous in the sense of (3), $|g(x)| \leq C_{g}\left(1+|x|^{p_{0}+1}\right)$. Then, we get $\left|g^{\varepsilon, N}(x)\right| \leq C_{g}\left(1+(N+1+\varepsilon)^{p_{0}+1}\right)$, and for $f$ being Lipschitz continuous in $y$ and $z$, uniformly in time, the same type of result applies.

(b) Since $\phi$ is a $C^{\infty}$ function and $f$ and $g$ are of polynomial growth, we get the result.

(c) Since $g$ is locally Hölder continuous, we get

$$
\begin{aligned}
& \left|g^{\varepsilon, N}(x)-g^{\varepsilon, N}(y)\right| \\
& \quad \leq \int_{-1}^{1}|\phi(u)| C_{g}\left(1+\left|b_{N}(x)-\varepsilon u\right|^{p_{0}}+\left|b_{N}(y)-\varepsilon u\right|^{p_{0}}\right)\left|b_{N}(x)-b_{N}(y)\right|^{\alpha} d u \\
& \quad \leq \int_{-1}^{1} C_{g}|\phi(u)|\left(1+(|x|+\varepsilon)^{p_{0}}+(|y|+\varepsilon)^{p_{0}}\right)|x-y|^{\alpha} d u \\
& \quad \leq C\left(C_{g}\right)\left(1+|x|^{p_{0}}+|y|^{p_{0}}\right)|x-y|^{\alpha} .
\end{aligned}
$$

(d) We have

$$
\left|g^{\varepsilon, N}(x)-g(x)\right|=\left|\int_{-1}^{1} \phi(u)\left(g\left(b_{N}(x)-\varepsilon u\right)-g(x)\right) d u\right|
$$




$$
\begin{aligned}
& \leq C_{g} \int_{-1}^{1}|\phi(u)|\left(1+\left|b_{N}(x)\right|^{p_{0}}+\varepsilon^{p_{0}}+|x|^{p_{0}}\right)\left(\left|b_{N}(x)-x\right|^{\alpha}+\varepsilon^{\alpha}\right) d u \\
& \leq C\left(C_{g}\right)\left(1+|x|^{p_{0}}\right)\left(\varepsilon^{\alpha}+|x|^{\alpha} \mathbf{1}_{|x| \geq N}\right),
\end{aligned}
$$

and the result follows.

(e) We simply have to apply the Lipschitz property of $f$ to get the result.

We put now $\varepsilon:=\frac{1}{N}$ and write $\left(g^{N}, f^{N}\right)$ instead of $\left(g^{\frac{1}{N}, N}, f^{\frac{1}{N}, N}\right)$ in order to simplify the notation and consider the BSDE

$$
Y_{t}^{N}=g^{N}\left(X_{T}\right)+\int_{t}^{T} f^{N}\left(r, Y_{r}^{N}, Z_{r}^{N}\right) d r-\int_{t}^{T} Z_{r}^{N} d B_{r}
$$

Representation for $\partial_{x}^{2} u^{N}(t, x)$

By (i) we have that

$$
u^{N}(t, x)=\mathbb{E}_{t, x} g^{N}\left(X_{T}^{t, x}\right)+\int_{t}^{T} \mathbb{E}_{t, x} f^{N}\left(r, Y_{r}^{N}, Z_{r}^{N}\right) d r
$$

According to Lemma 5.2 it holds that $\partial_{x}^{2} \mathbb{E}_{t, x} g^{N}\left(X_{T}\right)=\mathbb{E}_{t, x}\left[g^{N}\left(X_{T}\right) N_{T}^{t, 2}\right]$ and

$$
\partial_{x}^{2} \mathbb{E}_{t, x} f^{N}\left(r, Y_{r}^{N}, Z_{r}^{N}\right)=\mathbb{E}_{t, x}\left[f^{N}\left(r, Y_{r}^{N}, Z_{r}^{N}\right) N_{r}^{t, 2}\right]
$$

because

$$
f^{N}\left(r, Y_{r}^{N}, Z_{r}^{N}\right)=f^{N}\left(r, u^{N}\left(r, X_{r}\right), \sigma\left(r, X_{r}\right) u_{x}^{N}\left(r, X_{r}\right)\right)
$$

and $f^{N}(r, y, z)$ is continuous and bounded. Moreover, [24], Proposition 4 (or [21], Theorem 2.1) implies that $u^{N}(r, x)$ is $C^{1,2}$ and it holds that $\left|u^{N}(r, x)\right|+\left|\partial_{x} u^{N}(r, x)\right|+\left|\partial_{x}^{2} u^{N}(r, x)\right| \leq C^{N}$ for some $C^{N}>0$. Since $\sigma$ is continuous,

$$
(r, x) \mapsto f^{N}\left(r, u^{N}(r, x), \sigma(r, x) u_{x}^{N}(r, x)\right)
$$

is a bounded Borel function. Notice that by Lemma 5.2

$$
\mathbb{E}_{t, x}\left[N_{r}^{t, 2}\right]=0 \quad \text { and } \quad \mathbb{E}_{t, x}\left[\left(N_{r}^{t, 2}\right)^{2}\right] \leq \frac{\kappa_{2}^{2}}{(r-t)^{2}},
$$

so that

$$
\mathbb{E}_{t, x}\left[f^{N}\left(r, Y_{r}^{N}, Z_{r}^{N}\right) N_{r}^{t, 2}\right]=\mathbb{E}_{t, x}\left(\left[f^{N}\left(r, Y_{r}^{N}, Z_{r}^{N}\right)-f^{N}\left(r, Y_{t}^{N}, Z_{t}^{N}\right)\right] N_{r}^{t, 2}\right) .
$$


Using the Lipschitz continuity of $f^{N}$ (see Lemma 5.5), the inequality of Cauchy-Schwarz and Theorem 5.3 one can derive the upper bound

$$
\begin{aligned}
& \left|\partial_{x}^{2} \mathbb{E}_{t, x} f^{N}\left(r, Y_{r}^{N}, Z_{r}^{N}\right)\right| \\
& \quad \leq \mathbb{E}_{t, x}\left[\left|f^{N}\left(r, Y_{r}^{N}, Z_{r}^{N}\right)-f^{N}\left(r, Y_{t}^{N}, Z_{t}^{N}\right)\right|\left|N_{r}^{t, 2}\right|\right] \\
& \quad \leq C\left(L_{f}, \kappa_{2}\right)\left(\mathbb{E}_{t, x}\left(\left|Y_{r}^{N}-Y_{t}^{N}\right|^{2}+\left|Z_{r}^{N}-Z_{t}^{N}\right|^{2}\right)\right)^{\frac{1}{2}} \frac{1}{r-t} \\
& \quad \leq C\left(L_{f}, \kappa_{2}, C_{5.3}^{y}, C_{5.3}^{z}\right)\left[\left(\int_{t}^{r}(T-s)^{\alpha-1} d s\right)^{\frac{1}{2}}+\left(\int_{t}^{r}(T-s)^{\alpha-2} d s\right)^{\frac{1}{2}}\right] \frac{\Psi(x)}{r-t} \\
& \quad \leq C\left(T, L_{f}, \kappa_{2}, C_{5.3}^{y}, C_{5.3}^{z}\right) \Psi(x) \frac{1}{(T-r)^{1-\frac{\alpha}{2}}(r-t)^{\frac{1}{2}}} .
\end{aligned}
$$

By this we do have an integrable bound for the derivative, and by dominated convergence we get

$$
\begin{aligned}
\partial_{x}^{2} \int_{t}^{T} \mathbb{E}_{t, x} f^{N}\left(r, Y_{r}^{N}, Z_{r}^{N}\right) d r & =\int_{t}^{T} \partial_{x}^{2} \mathbb{E}_{t, x} f^{N}\left(r, Y_{r}^{N}, Z_{r}^{N}\right) d r \\
& =\int_{t}^{T} \mathbb{E}_{t, x}\left\{\left[f^{N}\left(r, Y_{r}^{N}, Z_{r}^{N}\right)-f^{N}\left(r, Y_{t}^{N} Z_{t}^{N}\right)\right] N_{r}^{t, 2}\right\} d r .
\end{aligned}
$$

Hence, we can write (using Fubini's theorem for the integral)

$$
\partial_{x}^{2} u^{N}(t, x)=\mathbb{E}_{t, x}\left(g^{N}\left(X_{T}\right) N_{T}^{t, 2}+\int_{t}^{T}\left[f^{N}\left(r, Y_{r}^{N}, Z_{r}^{N}\right)-f^{N}\left(r, Y_{t}^{N}, Z_{t}^{N}\right)\right] N_{r}^{t, 2} d r\right) .
$$

Convergence of $\partial_{x}^{2} u^{N}(t, x)$

Since $\mathbb{E}_{t, x}\left[\mathbb{E}_{t, x}\left(g^{N}\left(X_{T}\right)\right) N_{T}^{t, 2}\right]=0$, Cauchy-Schwarz's inequality and the local Hölder continuity of $g^{N}$ (see Lemma 5.5) give like in (24) that

$$
\begin{aligned}
\left|\mathbb{E}_{t, x}\left(g^{N}\left(X_{T}\right) N_{T}^{t, 2}\right)\right| & =\left|\mathbb{E}_{t, x}\left(\left[g^{N}\left(X_{T}\right)-\mathbb{E}_{t, x}\left(g^{N}\left(X_{T}\right)\right)\right] N_{T}^{t, 2}\right)\right| \\
& \leq\left(\mathbb{E}_{t, x}\left(\left|g^{N}\left(X_{T}\right)-\mathbb{E}_{t, x}\left(g^{N}\left(X_{T}\right)\right)\right|^{2}\right)\right)^{\frac{1}{2}} \frac{\kappa_{2}}{T-t} \\
& \leq C\left(C_{g}, T, p_{0}, \kappa_{2}, b, \sigma\right) \frac{\Psi(x)}{(T-t)^{1-\frac{\alpha}{2}}},
\end{aligned}
$$

for all $N \in \mathbb{N}$. For the second term we can use the upper bound (28) and Lemma A.2 to get

$$
\begin{aligned}
& \mathbb{E}_{t, x} \int_{t}^{T}\left|\left[f^{N}\left(r, Y_{r}^{N}, Z_{r}^{N}\right)-f^{N}\left(r, Y_{t}^{N}, Z_{t}^{N}\right)\right] N_{r}^{t, 2}\right| d r \\
& \quad \leq C\left(T, L_{f}, \kappa_{2}, C_{5.3}^{y}, C_{5.3}^{z}\right) \int_{t}^{T} \frac{\Psi(x)}{(T-r)^{1-\frac{\alpha}{2}}(r-t)^{\frac{1}{2}}} d r,
\end{aligned}
$$




$$
\leq C\left(T, L_{f}, \kappa_{2}, C_{5.3}^{y}, C_{5.3}^{z}\right) \Psi(x) \frac{B\left(\frac{\alpha}{2}, \frac{1}{2}\right)}{(T-t)^{\frac{1}{2}-\frac{\alpha}{2}}}
$$

which implies

$$
\left|\partial_{x}^{2} u^{N}(t, x)\right| \leq C\left(C_{g}, T, L_{f}, p_{0}, \kappa_{2}, C_{5.3}^{y}, C_{5.3}^{z}, b, \sigma\right) \frac{\Psi(x)}{(T-t)^{1-\frac{\alpha}{2}}} .
$$

According to [21], Theorem 2.1, $\partial_{x}^{2} u^{N}(t, x)$ is continuous. Let

$$
v(t, x):=\mathbb{E}_{t, x}\left(g\left(X_{T}\right) N_{T}^{t, 2}+\int_{t}^{T}\left[f\left(r, Y_{r}, Z_{r}\right)-f\left(r, Y_{t}, Z_{t}\right)\right] N_{r}^{t, 2} d r\right) .
$$

We show that for any $(t, x) \in[0, T) \times \mathbb{R}$ it holds $\partial_{x}^{2} u^{N}(t, x) \rightarrow v(t, x)$ if $N \rightarrow \infty$, and that $v$ is continuous on $[0, T) \times \mathbb{R}$. The idea to show continuity of $v$ is as follows: If $\left(t_{n}, x_{n}\right) \rightarrow(t, x)$, then we may assume that we can find a $\delta>0$ such that $x_{n} \in(x-\delta, x+\delta)$ and $t_{n} \in(t-\delta, t+\delta) \subseteq$ $[0, T)$ for each sufficiently large $n$. We consider

$$
\begin{aligned}
\left|v\left(t_{n}, x_{n}\right)-v(t, x)\right| \leq & \left|v\left(t_{n}, x_{n}\right)-\partial_{x}^{2} u^{N}\left(t_{n}, x_{n}\right)\right|+\left|\partial_{x}^{2} u^{N}\left(t_{n}, x_{n}\right)-\partial_{x}^{2} u^{N}(t, x)\right| \\
& +\left|\partial_{x}^{2} u^{N}(t, x)-v(t, x)\right| .
\end{aligned}
$$

Since $\partial_{x}^{2} u^{N}$ is continuous, the term $\left|\partial_{x}^{2} u^{N}\left(t_{n}, x_{n}\right)-\partial_{x}^{2} u^{N}(t, x)\right|$ is small for large $n$. Hence, it suffices to show that $\sup _{s \in(t-\delta, t+\delta), y \in(x-\delta, x+\delta)}\left|\partial_{x}^{2} u^{N}(s, y)-v(s, y)\right|$ is small for large $N$. Let $(s, y) \in(t-\delta, t+\delta) \times(x-\delta, x+\delta)$. It holds

$$
\left|\partial_{x}^{2} u^{N}(s, y)-v(s, y)\right| \leq \mathbb{E}_{s, y}\left|\left[g^{N}\left(X_{T}\right)-g\left(X_{T}\right)\right] N_{T}^{s, 2}\right|+\int_{s}^{T} D^{\frac{1}{2}}(r, s) \frac{\kappa_{2}}{r-s} d r:=D_{1}+D_{2},
$$

where (setting $\left.\|\cdot\|_{\mathbb{P}_{s, y}}:=\|\cdot\|_{L_{2}\left(\mathbb{P}_{s, y}\right)}\right)$

$$
\begin{aligned}
D(r, s):= & \left\|f^{N}\left(r, Y_{r}^{N}, Z_{r}^{N}\right)-f^{N}\left(r, Y_{s}^{N}, Z_{s}^{N}\right)-\left[f\left(r, Y_{r}, Z_{r}\right)-f\left(r, Y_{s}, Z_{s}\right)\right]\right\|_{\mathbb{P}_{s, y}}^{2} \\
\leq & L_{f}\left(\left\|Y_{r}^{N}-Y_{s}^{N}\right\|_{\mathbb{P}_{s, y}}+\left\|Z_{r}^{N}-Z_{s}^{N}\right\|_{\mathbb{P}_{s, y}}+\left\|Y_{r}-Y_{s}\right\|_{\mathbb{P}_{s, y}}+\left\|Z_{r}-Z_{s}\right\|_{\mathbb{P}_{s, y}}\right) \\
& \times\left(\left\|f^{N}\left(r, Y_{r}^{N}, Z_{r}^{N}\right)-f\left(r, Y_{r}, Z_{r}\right)\right\|_{\mathbb{P}_{s, y}}+\left\|f^{N}\left(r, Y_{s}^{N}, Z_{s}^{N}\right)-f\left(r, Y_{s}, Z_{s}\right)\right\|_{\mathbb{P}_{s, y}}\right) .
\end{aligned}
$$

First, let us bound $D_{1}$. According to Cauchy-Schwarz's inequality, (31) below and (27) we get

$$
D_{1} \leq \delta_{1} \sqrt{\mathbb{E}_{s, y}\left(\left|N_{T}^{s, 2}\right|^{2}\right)} \leq \frac{\delta_{1} \kappa_{2}}{T-s} \leq \frac{\delta_{1} \kappa_{2}}{T-t-\delta} .
$$

Now let us bound $D_{2}$. According to Theorem 5.3 it holds

$$
\begin{aligned}
D^{\frac{1}{2}}(r, s) \leq & C\left(T, L_{f}, C_{5.3}^{y}, C_{5.3}^{z}\right) \Psi^{\frac{1}{2}}(y) \frac{(r-s)^{\frac{1}{4}}}{(T-r)^{\frac{1}{2}-\frac{\alpha}{4}}} \\
& \times\left(\left\|f^{N}\left(r, Y_{r}^{N}, Z_{r}^{N}\right)-f\left(r, Y_{r}, Z_{r}\right)\right\|_{\mathbb{P}_{s, y}}+\left\|f^{N}\left(r, Y_{s}^{N}, Z_{s}^{N}\right)-f\left(r, Y_{s}, Z_{s}\right)\right\|_{\mathbb{P}_{s, y}}\right)^{\frac{1}{2}} .
\end{aligned}
$$


Then, using (32), (34), (35) and Proposition 5.6 below gives

$$
D^{\frac{1}{2}}(r, s) \leq C\left(T, L_{f}, \kappa_{2}, C_{5.3}^{y}, C_{5.3}^{z}\right) \Psi(y) \frac{(r-s)^{\frac{1}{4}}}{(T-r)^{\frac{1}{2}-\frac{\alpha}{4}}} \frac{\delta_{1}}{(T-r)^{\frac{1}{4}}} .
$$

Hence, we have shown that

$$
\begin{aligned}
D_{2} \leq & C\left(T, L_{f}, \kappa_{2}, C_{5.3}^{y}, C_{5.3}^{z}\right) \Psi(y) \int_{s}^{T} \frac{\delta_{1}}{(r-s)^{\frac{3}{4}}(T-r)^{\frac{1}{2}-\frac{\alpha}{4}+\frac{1}{4}}} d r \\
\leq & C\left(T, L_{f}, \kappa_{2}, C_{5.3}^{y}, C_{5.3}^{z}\right) \Psi(y) \frac{\delta_{1}}{(T-s)^{\frac{1}{2}-\frac{\alpha}{4}}}, \\
\leq & C\left(T, L_{f}, \kappa_{2}, C_{5.3}^{y}, C_{5.3}^{z}\right) \Psi(x+\delta) \frac{\delta_{1}}{(T-t-\delta)^{\frac{1}{2}-\frac{\alpha}{4}}} \\
& \forall(s, y) \in(t-\delta, t+\delta) \times(x-\delta, x+\delta) .
\end{aligned}
$$

Consequently, $\sup _{y \in(x-\delta, x+\delta), s \in(t-\delta, t+\delta)}\left|\partial_{x}^{2} u^{N}(s, y)-v(s, y)\right|$ is small for large $N$, hence $v$ is continuous. Since

$$
\partial_{x} u^{N}(t, x)-\partial_{x} u^{N}(t, y)=\int_{y}^{x} \partial_{x}^{2} u^{N}(t, z) d z
$$

converges to

$$
\partial_{x} u(t, x)-\partial_{x} u(t, y)=\int_{y}^{x} v(t, z) d z
$$

it follows that $\partial_{x}^{2} u(t, x)=v(t, x)$. Then point (iii-a) and (23) are proved. Since $\partial_{x}^{2} u^{N}$ converges to $v$ for $N \rightarrow \infty$, we deduce point (iii-b) from (29).

Proposition 5.6. Let Assumptions 5.1 and 2.1 hold. Then for any $(s, y) \in(t-\delta, t+\delta) \times(x-$ $\delta, x+\delta$ ) with $t+\delta<T$ and $r$ such that $s \leq r<T$ we have

$$
\left\|Y_{r}^{N}-Y_{r}\right\|_{L_{2}\left(\mathbb{P}_{s, y}\right)}+\left\|Z_{r}^{N}-Z_{r}\right\|_{L_{2}\left(\mathbb{P}_{s, y}\right)} \leq \frac{\delta_{1}}{\sqrt{T-r}},
$$

where $\delta_{1}$ denotes a generic constant which tends to 0 when $N$ tends to $+\infty$.

Proof. Let here $\|\cdot\|$ stand for $\|\cdot\|_{L_{2}\left(\mathbb{P}_{s, y}\right)}$. We will use for the $Y$ differences the inequality

$$
\left\|Y_{r}^{N}-Y_{r}\right\| \leq\left\|g^{N}\left(X_{T}\right)-g\left(X_{T}\right)\right\|+\int_{r}^{T}\left\|f^{N}\left(w, Y_{w}^{N}, Z_{w}^{N}\right)-f\left(w, Y_{w}, Z_{w}\right)\right\| d w
$$


For the $Z$ differences, we get by (22) and (ii-b)

$$
\begin{aligned}
\left\|Z_{r}^{N}-Z_{r}\right\| & \\
\leq & C(\sigma)\left(\left\|\mathbb{E}_{r}\left(g^{N}\left(X_{T}\right)-g\left(X_{T}\right)\right) N_{T}^{r, 1}\right\|\right. \\
& \left.+\left\|\mathbb{E}_{r} \int_{r}^{T}\left(f^{N}\left(w, Y_{w}^{N}, Z_{w}^{N}\right)-f\left(w, Y_{w}, Z_{w}\right)\right) N_{w}^{r, 1} d w\right\|\right) \\
\leq & C\left(\kappa_{2}, \sigma\right)\left(\frac{\left\|g^{N}\left(X_{T}\right)-g\left(X_{T}\right)\right\|}{\sqrt{T-r}}+\int_{r}^{T}\left\|f^{N}\left(w, Y_{w}^{N}, Z_{w}^{N}\right)-f\left(w, Y_{w}, Z_{w}\right)\right\| \frac{1}{\sqrt{w-r}} d w\right) .
\end{aligned}
$$

Let $S(r):=\left\|Y_{r}^{N}-Y_{r}\right\|+\left\|Z_{r}^{N}-Z_{r}\right\|$. Using the inequality $\left(1+\frac{1}{\sqrt{w-r}}\right) \leq C(T) \frac{1}{\sqrt{w-r}}$ for $r<$ $w \leq T$ gives

$$
\begin{aligned}
S(r) \leq & C\left(T, \kappa_{2}, \sigma\right)\left(\left\|g^{N}\left(X_{T}\right)-g\left(X_{T}\right)\right\| \frac{1}{\sqrt{T-r}}\right. \\
& \left.+\int_{r}^{T}\left\|f^{N}\left(w, Y_{w}^{N}, Z_{w}^{N}\right)-f\left(w, Y_{w}, Z_{w}\right)\right\| \frac{1}{\sqrt{w-r}} d w\right) .
\end{aligned}
$$

Let us bound $\left\|g^{N}\left(X_{T}\right)-g\left(X_{T}\right)\right\|$. By Lemma 5.5, we get the estimate

$$
\begin{aligned}
\mathbb{E}_{s, y}\left|g^{N}\left(X_{T}\right)-g\left(X_{T}\right)\right|^{2} & \leq C\left(C_{g}\right) \mathbb{E}_{s, y}\left(\Psi\left(X_{T}\right)^{4}\right)^{\frac{1}{2}}\left(\mathbb{E}_{s, y}\left(\frac{1}{N^{\alpha}}+\frac{\left|X_{T}\right|^{\alpha+1}}{N}\right)^{4}\right)^{\frac{1}{2}} \\
& \leq C\left(C_{g}, T, b, \sigma, p_{0}\right) \Psi(y)^{2}\left(\frac{1}{N^{2 \alpha}}+\frac{|y|^{2 \alpha+2}}{N^{2}}\right) \\
& \leq C\left(C_{g}, T, b, \sigma, p_{0}\right) \Psi(x+\delta)^{2}\left(\frac{1}{N^{2 \alpha}}+\frac{|x+\delta|^{2 \alpha+2}}{N^{2}}\right) \leq \delta_{1}^{2},
\end{aligned}
$$

for any arbitrarily small $\delta_{1}>0$, provided that $N$ is sufficiently large. Let us now bound $\left\|f^{N}\left(w, Y_{w}^{N}, Z_{w}^{N}\right)-f\left(w, Y_{w}, Z_{w}\right)\right\|$. Using again Lemma 5.5 yields to

$$
\begin{aligned}
& \left\|f^{N}\left(w, Y_{w}^{N}, Z_{w}^{N}\right)-f\left(w, Y_{w}, Z_{w}\right)\right\| \\
& \quad \leq\left\|f^{N}\left(w, Y_{w}^{N}, Z_{w}^{N}\right)-f^{N}\left(w, Y_{w}, Z_{w}\right)\right\|+\left\|f^{N}\left(w, Y_{w}, Z_{w}\right)-f\left(w, Y_{w}, Z_{w}\right)\right\| \\
& \quad \leq L_{f}\left(\left\|Y_{w}^{N}-Y_{w}\right\|+\left\|Z_{w}^{N}-Z_{w}\right\|+\frac{2}{N}+\left\|b_{N}\left(Y_{w}\right)-Y_{w}\right\|+\left\|b_{N}\left(Z_{w}\right)-Z_{w}\right\|\right) .
\end{aligned}
$$


Then, plugging (31) and (32) into (30) gives

$$
\begin{aligned}
S(r) \leq & \frac{C\left(T, \kappa_{2}, \sigma\right) \delta_{1}}{\sqrt{T-r}}+C\left(T, \kappa_{2}, \sigma\right) L_{f} \int_{r}^{T} \frac{S(w)}{\sqrt{w-r}} d w \\
& +C\left(T, \kappa_{2}, \sigma\right) L_{f} \int_{r}^{T} \frac{\frac{1}{N}+\left\|b_{N}\left(Y_{w}\right)-Y_{w}\right\|+\left\|b_{N}\left(Z_{w}\right)-Z_{w}\right\|}{\sqrt{w-r}} d w .
\end{aligned}
$$

To estimate $\left\|b_{N}\left(Z_{w}\right)-Z_{w}\right\|$ we use $Z_{w}=\sigma\left(w, X_{w}\right) u_{x}\left(w, X_{w}\right)$ and choose a small $a>0$ such that $\beta:=\frac{(2+a)(1-\alpha)}{2}<1$. Then

$$
\begin{aligned}
\left\|b_{N}\left(Z_{w}\right)-Z_{w}\right\|^{2} & =\mathbb{E}_{s, y}\left|b_{N}\left(Z_{w}\right)-Z_{w}\right|^{2} \mathbf{1}_{\left|Z_{w}\right| \geq N} \\
& \leq \frac{\mathbb{E}_{s, y}\left|Z_{w}\right|^{2+a}}{N^{a}}=\frac{\mathbb{E}_{s, y}\left|\sigma\left(w, X_{w}\right) u_{x}\left(w, X_{w}\right)\right|^{2+a}}{N^{a}} .
\end{aligned}
$$

Using Theorem 5.4(ii-c) yields

$$
\begin{aligned}
\mathbb{E}_{s, y}\left|b_{N}\left(Z_{w}\right)-Z_{w}\right|^{2} & \leq \frac{C\left(c_{5.4}^{2}, \sigma\right) \mathbb{E}_{s, y} \Psi\left(X_{w}\right)^{(2+a)}}{(T-w)^{\frac{(2+a)(1-\alpha)}{2}} N^{a}} \leq \frac{C\left(T, p_{0}, c_{5.4}^{2}, \sigma, b\right) \Psi(y)^{(2+a)}}{(T-w)^{\frac{(2+a)(1-\alpha)}{2}} N^{a}} \\
& \leq \frac{\delta_{1}}{(T-w)^{\frac{(2+a)(1-\alpha)}{2}}}, \quad \forall(s, y) \in(t-\delta, t+\delta) \times(x-\delta, x+\delta) .
\end{aligned}
$$

Similarly,

$$
\begin{aligned}
\mathbb{E}_{s, y}\left|b_{N}\left(Y_{w}\right)-Y_{w}\right|^{2} & \leq \frac{C\left(T, p_{0}, c_{5.4}^{1}, b, \sigma\right) \Psi(y)^{(2+a)}}{N^{a}} \\
& \leq \delta_{1}, \quad \forall(s, y) \in(t-\delta, t+\delta) \times(x-\delta, x+\delta) .
\end{aligned}
$$

Plugging (34) and (35) into (33) gives

$$
\begin{aligned}
S(r) \leq & \frac{C\left(T, \kappa_{2}\right) \delta_{1}}{\sqrt{T-r}}+C\left(T, \kappa_{2}\right) L_{f} \int_{r}^{T} \frac{S(w)}{\sqrt{w-r}} d w \\
& +C\left(T, \kappa_{2}\right) L_{f} \int_{r}^{T} \frac{\frac{1}{N}+\delta_{1}}{\sqrt{w-r}}+\frac{\delta_{1}}{(T-w)^{\frac{(2+a)(1-\alpha)}{2}} \sqrt{w-r}} d w \\
\leq & C\left(T, \kappa_{2}, L_{f}\right)\left(\frac{\delta_{1}}{\sqrt{T-r}}+\int_{r}^{T} \frac{S(w)}{\sqrt{w-r}} d w\right),
\end{aligned}
$$

where the last inequality comes from Lemma A.2 $(\beta<1)$. If we iterate this inequality by replacing $S(w)$ with its estimate and then change the oder of integration we get by Lemma A.2 that

$$
S(r) \leq C\left(T, \kappa_{2}, L_{f}\right)
$$




$$
\begin{aligned}
& \times\left(\frac{\delta_{1}}{\sqrt{T-r}}+\int_{r}^{T} \frac{\delta_{1}}{\sqrt{T-w}} \frac{1}{\sqrt{w-r}} d w+\int_{r}^{T} \int_{w}^{T} \frac{S(v)}{\sqrt{v-w} \sqrt{w-r}} d v d w\right) \\
\leq & C\left(T, \kappa_{2}, L_{f}\right)\left(\frac{\delta_{1}}{\sqrt{T-r}}+\delta_{1} B\left(\frac{1}{2}, \frac{1}{2}\right)+B\left(\frac{1}{2}, \frac{1}{2}\right) \int_{r}^{T} S(v) d v\right) .
\end{aligned}
$$

It remains to apply Gronwall's lemma to see that $S(r) \leq \frac{C\left(T, \kappa_{2}, L_{f}\right) \delta_{1}}{\sqrt{T-r}}$. Since $C\left(T, \kappa_{2}, L_{f}\right) \delta_{1}$ becomes arbitrarily small for $N$ large, we will slightly abuse the notation and write $S(r) \leq \frac{\delta_{1}}{\sqrt{T-r}}$.

\section{Appendix: Technical results and estimates}

Lemma A.1. For all $0 \leq k \leq m \leq n$ and $p>0$, it holds for $h=\frac{T}{n}$ that

(i) $\mathbb{E} \tau_{k}=k h$,

(ii) $\mathbb{E}\left|\tau_{1}\right|^{p} \leq C(p) h^{p}$,

(iii) $\mathbb{E}\left|B_{\tau_{m}}-B_{\tau_{k}}\right|^{2}=t_{m}-t_{k}$,

(iv) $\mathbb{E}\left|B_{\tau_{k}}-B_{t_{k}}\right|^{2 p} \leq C(p) \mathbb{E}\left|\tau_{k}-t_{k}\right|^{p} \leq C(p)\left(t_{k} h\right)^{\frac{p}{2}}$.

Proof. The strong Markov property of the Brownian motion implies that $\left(\tau_{i}-\tau_{i-1}\right)_{i=1}^{\infty}$ is an i.i.d. sequence. According to [33], Proposition 11.1(iii), we have that $\mathbb{E} \tau_{1}=\frac{T}{n}$, and (i) follows. Item (ii) follows by [33], Proposition 11.1(iv), and Jensen's inequality. To prove item (iii), recall that $\left(B_{\tau_{i}}-B_{\tau_{i-1}}\right)_{i=1}^{\infty}$ is a centered i.i.d. sequence with $\mathbb{E}\left(B_{\tau_{i}}-B_{\tau_{i-1}}\right)^{2}=h, i \geq 1$. (iv): The BDG inequality implies that for each $p>0$,

$$
\begin{aligned}
\mathbb{E}\left|B_{\tau_{k}}-B_{t_{k}}\right|^{p} & =\mathbb{E}\left|\int_{0}^{\tau_{k} \vee t_{k}}\left(\mathbf{1}_{\left[0, \tau_{k}\right]}(r)-\mathbf{1}_{\left[0, t_{k}\right]}(r)\right) d B_{r}\right|^{p} \\
& \leq C(p) \mathbb{E}\left(\int_{0}^{\tau_{k} \vee t_{k}} \mathbf{1}_{\left[0, \tau_{k}\right] \Delta\left[0, t_{k}\right]}(r) d r\right)^{p / 2}=\mathbb{E}\left|\tau_{k}-t_{k}\right|^{p / 2} .
\end{aligned}
$$

To prove the second inequality of (iv), a generalization of [33], Proposition 11.1(iv), we first assume that $p \geq 1$. Let us rewrite $\tau_{k}-t_{k}=\sum_{i=1}^{k} \eta_{i}$ where $\left(\eta_{i}\right)_{1 \leq i \leq k}$ is an i.i.d. centered sequence of random variables distributed as $\tau_{1}-h$. Burkholder's and Hölder's inequalities, and finally item (ii) yield

$$
\mathbb{E}\left|\tau_{k}-t_{k}\right|^{p} \leq C(p) \mathbb{E}\left(\sum_{i=1}^{k} \eta_{i}^{2}\right)^{\frac{p}{2}} \leq k^{\frac{p}{2}-1} \sum_{i=1}^{k} \mathbb{E}\left(\eta_{i}^{p}\right) \leq C(p)\left(t_{k} h\right)^{\frac{p}{2}},
$$

which proves the claim for $p \geq 1$. The case $p<1$ follows from this result by Jensen's inequality.

Lemma A.2. For all $t \in[0, T)$ and for all $\alpha<1, \beta<1$ we have

$$
\int_{t}^{T} \frac{1}{(T-r)^{\alpha}(r-t)^{\beta}} d r=\frac{1}{(T-t)^{\alpha+\beta-1}} B(1-\alpha, 1-\beta),
$$

where $B$ denotes the beta function. 


\section{References}

[1] Alanko, S. (2015). Regression-based Monte Carlo methods for solving nonlinear PDEs. Ph.D. thesis, New York University.

[2] Bally, V. (1997). Approximation scheme for solutions of BSDE. In Backward Stochastic Differential Equations (Paris, 1995-1996). Pitman Res. Notes Math. Ser. 364 177-191. Harlow: Longman. MR1752682

[3] Bally, V. and Pagès, G. (2003). A quantization algorithm for solving multi-dimensional discretetime optimal stopping problems. Bernoulli 9 1003-1049. MR2046816 https://doi.org/10.3150/bj/ 1072215199

[4] Bender, C. and Zhang, J. (2008). Time discretization and Markovian iteration for coupled FBSDEs. Ann. Appl. Probab. 18 143-177. MR2380895 https://doi.org/10.1214/07-AAP448

[5] Borodin, A.N. and Salminen, P. (2015). Handbook of Brownian Motion: Facts and Formulae, 2nd ed. Probability and Its Applications. Basel: Birkhäuser.

[6] Bouchard, B. and Touzi, N. (2004). Discrete-time approximation and Monte-Carlo simulation of backward stochastic differential equations. Stochastic Process. Appl. 111 175-206. MR2056536 https://doi.org/10.1016/j.spa.2004.01.001

[7] Briand, Ph., Delyon, B. and Mémin, J. (2001). Donsker-type theorem for BSDEs. Electron. Commun. Probab. 6 1-14. MR1817885 https://doi.org/10.1214/ECP.v6-1030

[8] Briand, Ph., Delyon, B., Hu, Y., Pardoux, E. and Stoica, L. (2003). $L^{p}$ solutions of backward stochastic differential equations. Stochastic Process. Appl. 108 109-129. MR2008603 https://doi.org/10. 1016/S0304-4149(03)00089-9

[9] Briand, Ph. and Labart, C. (2014). Simulation of BSDEs by Wiener chaos expansion. Ann. Appl. Probab. 24 1129-1171. MR3199982 https://doi.org/10.1214/13-AAP943

[10] Chassagneux, J.-F. (2014). Linear multistep schemes for BSDEs. SIAM J. Numer. Anal. 52 2815-2836. MR3284573 https://doi.org/10.1137/120902951

[11] Chassagneux, J.-F. and Crisan, D. (2014). Runge-Kutta schemes for backward stochastic differential equations. Ann. Appl. Probab. 24 679-720. MR3178495 https://doi.org/10.1214/13-AAP933

[12] Chassagneux, J.-F., Crisan, D. and Delarue, F. (2017). Numerical method for FBSDEs of McKeanVlasov type. Available at arXiv:1703.02007.

[13] Chassagneux, J.-F. and Garcia Trillos, C.A. (2017). Cubature methods to solve BSDEs: Error expansion and complexity control. Available at arXiv:1702.00999.

[14] Chassagneux, J.-F. and Richou, A. (2015). Numerical stability analysis of the Euler scheme for BSDEs. SIAM J. Numer. Anal. 53 1172-1193. MR3338675 https://doi.org/10.1137/140977047

[15] Chassagneux, J.-F. and Richou, A. (2016). Rate of convergence for discrete-time approximation of reflected BSDEs arising in switching problems. Available at arXiv:1602.00015.

[16] Chaudru de Raynal, P.E. and Garcia Trillos, C.A. (2015). A cubature based algorithm to solve decoupled McKean-Vlasov forward-backward stochastic differential equations. Stochastic Process. Appl. 125 2206-2255. MR3322862 https://doi.org/10.1016/j.spa.2014.11.018

[17] Chevance, D. (1997). Résolution numérique des équations différentielles stochastiques rétrogrades. $\mathrm{Ph} . \mathrm{D}$. thesis, Université de Provence-Aix-Marseille I, Marseille.

[18] Coquet, F., Mackevičius, V. and Mémin, J. (1999). Corrigendum to: "Stability in $\mathbb{D}$ of martingales and backward equations under discretization of filtration" [Stochastic Processes Appl. 75 (1998), no. 2, 235-248; MR1632205 (99f:60109)]. Stochastic Process. Appl. 82 335-338. MR1700013 https://doi.org/10.1016/S0304-4149(99)00020-4

[19] Crisan, D. and Delarue, F. (2012). Sharp derivative bounds for solutions of degenerate semi-linear partial differential equations. J. Funct. Anal. 263 3024-3101. MR2973334 https://doi.org/10.1016/j. jfa.2012.07.015 
[20] Crisan, D., Manolarakis, K. and Touzi, N. (2010). On the Monte Carlo simulation of BSDEs: An improvement on the Malliavin weights. Stochastic Process. Appl. 120 1133-1158. MR2639741 https://doi.org/10.1016/j.spa.2010.03.015

[21] Delarue, F. and Menozzi, S. (2006). A forward-backward stochastic algorithm for quasi-linear PDEs. Ann. Appl. Probab. 16 140-184. MR2209339 https://doi.org/10.1214/105051605000000674

[22] Geiss, C., Geiss, S. and Gobet, E. (2012). Generalized fractional smoothness and $L_{p}$-variation of BSDEs with non-Lipschitz terminal condition. Stochastic Process. Appl. 122 2078-2116. MR2921973 https://doi.org/10.1016/j.spa.2012.02.006

[23] Geiss, C. and Labart, C. (2016). Simulation of BSDEs with jumps by Wiener chaos expansion. Stochastic Process. Appl. 126 2123-2162. MR3483749 https://doi.org/10.1016/j.spa.2016.01.006

[24] Gobet, E. and Labart, C. (2007). Error expansion for the discretization of backward stochastic differential equations. Stochastic Process. Appl. 117 803-829. MR2330720 https://doi.org/10.1016/j.spa. 2006.10.007

[25] Gobet, E., Lemor, J.-P. and Warin, X. (2005). A regression-based Monte Carlo method to solve backward stochastic differential equations. Ann. Appl. Probab. 15 2172-2202. MR2152657 https://doi.org/10.1214/105051605000000412

[26] Henry-Labordère, P., Tan, X. and Touzi, N. (2014). A numerical algorithm for a class of BSDEs via the branching process. Stochastic Process. Appl. 124 1112-1140. MR3138609 https://doi.org/10.1016/j. spa.2013.10.005

[27] Ma, J., Protter, P., San Martín, J. and Torres, S. (2002). Numerical method for backward stochastic differential equations. Ann. Appl. Probab. 12 302-316. MR1890066 https://doi.org/10.1214/aoap/ 1015961165

[28] Ma, J. and Zhang, J. (2002). Representation theorems for backward stochastic differential equations. Ann. Appl. Probab. 12 1390-1418. MR1936598 https://doi.org/10.1214/aoap/1037125868

[29] Martínez, M., San Martín, J. and Torres, S. (2011). Numerical method for reflected backward stochastic differential equations. Stoch. Anal. Appl. 29 1008-1032. MR2847333 https://doi.org/10.1080/ 07362994.2011 .610162

[30] Mémin, J., Peng, S. and Xu, M. (2008). Convergence of solutions of discrete reflected backward SDE's and simulations. Acta Math. Appl. Sin. Engl. Ser. 24 1-18. MR2385005 https://doi.org/10. 1007/s10255-006-6005-6

[31] Peng, S. and Xu, M. (2011). Numerical algorithms for backward stochastic differential equations with 1-D Brownian motion: Convergence and simulations. ESAIM Math. Model. Numer. Anal. 45 335-360. MR2804642 https://doi.org/10.1051/m2an/2010059

[32] Sundar, P. and Yin, H. (2009). Existence and uniqueness of solutions to the backward 2D stochastic Navier-Stokes equations. Stochastic Process. Appl. 119 1216-1234. MR2508571 https://doi.org/10. 1016/j.spa.2008.06.007

[33] Walsh, J.B. (2003). The rate of convergence of the binomial tree scheme. Finance Stoch. 7 337-361. MR1994913 https://doi.org/10.1007/s007800200094

[34] Weinan, E., Hutzenthaler, M., Jentzen, A. and Kruse, T. (2017). On multilevel Picard numerical approximations for high-dimensional nonlinear parabolic partial differential equations and highdimensional nonlinear backward stochastic differential equations. Available at arXiv:1708.03223.

[35] Zhang, J. (2001). Some fine properties of backward stochastic differential equations, with applications. $\mathrm{Ph} . \mathrm{D}$. thesis, Purdue University.

[36] Zhang, J. (2004). A numerical scheme for BSDEs. Ann. Appl. Probab. 14 459-488. MR2023027 https://doi.org/10.1214/aoap/1075828058

[37] Zhang, J. (2005). Representation of solutions to BSDEs associated with a degenerate FSDE. Ann. Appl. Probab. 15 1798-1831. MR2152246 https://doi.org/10.1214/105051605000000232

Received September 2018 and revised February 2019 